\title{
C9orf72 is differentially expressed in the central nervous system and myeloid cells and consistently reduced in C9orf72, MAPT and GRN mutation carriers
}

Patrizia Rizzu ${ }^{1 *+}$, Cornelis Blauwendraat ${ }^{1 \dagger}$, Sasja Heetveld ${ }^{1+}$, Emily M. Lynes ${ }^{1}$, Melissa Castillo-Lizardo ${ }^{1}$, Ashutosh Dhingra ${ }^{1}$, Elwira Pyz ${ }^{1}$, Markus Hobert ${ }^{1,2}$, Matthis Synofzik ${ }^{1,2}$, Javier Simón-Sánchez ${ }^{1,2}$,

Margherita Francescatto ${ }^{1}$ and Peter Heutink ${ }^{1,2}$

\begin{abstract}
A non-coding hexanucleotide repeat expansion (HRE) in C9orf72 is a common cause of amyotrophic lateral sclerosis (ALS) and frontotemporal dementia (FTD) acting through a loss of function mechanism due to haploinsufficiency of C9orf72 or a gain of function mediated by aggregates of bidirectionally transcribed HRE-RNAs translated into dipeptide repeat (DPR) proteins. To fully understand regulation of C9orf72 expression we surveyed the C9orf72 locus using Cap Analysis of Gene Expression sequence data (CAGEseq). We observed C9orf72 was generally lowly expressed with the exception of a subset of myeloid cells, particularly CD14+ monocytes that showed up to seven fold higher expression as compared to central nervous system (CNS) and other tissues. The expression profile at the C9orf72 locus showed a complex architecture with differential expression of the transcription start sites (TSSs) for the annotated C9orf72 transcripts between myeloid and CNS tissues suggesting cell and/or tissue specific functions. We further detected novel TSSs in both the sense and antisense strand at the C9orf72 locus and confirmed their existence in brain tissues and CD14+ monocytes. Interestingly, our experiments showed a consistent decrease of C9orf72 coding transcripts not only in brain tissue and monocytes from C9orf72-HRE patients, but also in brains from MAPT and GRN mutation carriers together with an increase in antisense transcripts suggesting these could play a role in regulation of C9orf72. We found that the non-HRE related expression changes cannot be explained by promoter methylation but by the presence of the C9orf72-HRE risk haplotype and unknown functional interactions between C9orf72, MAPT and GRN.
\end{abstract}

Keywords: Neurodegeneration, C9orf72 risk haplotype, Hexanucleotide repeat expansion, Frontotemporal dementia

\section{Introduction}

In 2011 two independent studies identified a noncoding HRE in the C9orf72 gene as the major cause for chromosome 9-linked ALS and FTD with or without concomitant motor neuron disease $[1,2]$. Since then, rapid progress has been made in elucidating the pathological and mechanistic aspects of the disease causing mutation. The current hypotheses suggest that the

\footnotetext{
* Correspondence: patrizia.rizzu@dzne.de

${ }^{\dagger}$ Equal contributors

${ }^{1}$ German Center for Neurodegenerative Diseases (DZNE), Otfried-Müller

Strasse 23, Tübingen 72076, Germany

Full list of author information is available at the end of the article
}

disease occurs through, not necessarily exclusive, lossand gain of toxicity function mechanisms mediated by (1) haploinsufficiency, (2) transcription of sense and antisense HRE-RNAs and (3) translation of these RNAs into DPR proteins through unconventional repeatassociated non-ATG (RAN) translation $[3,4]$.

Although the exact pathogenic mechanisms are not yet fully understood, the repeat-mediated toxicity hypothesis is gaining momentum. Bidirectionally transcribed HRE containing RNAs accumulate into RNA foci, occurring mainly in the nuclei of neurons in brain tissue and cultured cells of patients $[1,5,6]$. HRE-RNA transcripts can form hairpin and G-quadruplex structures $[7,8]$ and 
induce a toxic RNA gain of function by binding and sequestering RNA-binding proteins involved in splicing [9] and nucleocytoplasmic trafficking $[10,11]$ consequently altering their availability for their normal function.

The C9orf72 DPR proteins accumulate into cytoplasmic and intranuclear inclusions in brains of patients [12-16]. And studies in cell culture and animal models strongly corroborate that overexpression of DPR proteins is toxic and can induce nuclear inclusions and nucleolar stress $[17,18]$.

A loss of function mechanism for C9orf 72 has also been suggested, based on the observed decrease in C9orf72 mRNA expression in brain tissue and iPSC derived-neurons of C9orf72-HRE patients. This reduction in expression could be mediated by the Gquadruplex structures formed by the HRE-RNAs, which would impair C9orf72 full-length transcription as a result of the repeat-length dependent accumulation of aborted transcripts of C9orf72 [17].

Further evidence for this hypothesis comes from the targeted reduction of the C9orf72 orthologue in zebrafish that resulted in axonopathy and motor deficits and from a C.elegans C9orf72 knockout model that presented with motor phenotypes, suggesting that loss of C9orf72 protein can lead to motor deficits $[19,20]$. However, silencing of $C 9$ orf 72 by intracerebroventricular delivery of antisense oligonucleotides in adult mice or by neuralspecific ablation in conditional C9orf 72 knock-out mice $[5,21]$ did not lead to motor or behavioral phenotype arguing against a loss of function as the primary pathogenic mechanism. But even though $C 9$ orf 72 reduction might not be the major culprit, it could still be detrimental to cells as substantial evidence supports $C 9$ orf 72 interrelated roles in protein trafficking [22-24] and autophagy [25]. In this context and considering possible therapeutic approaches, it becomes important to fully understand how C9orf72 RNA expression is regulated. Up until now, the attention of the field has been mainly focused on the repeat expansion and the immediate neighboring sequence, with several studies suggesting that epigenetic changes like the C9orf72 promoter hypermethylation, might partially contribute to transcriptional silencing of mutant C9orf72 [26, 27]. To help understand additional mechanisms contributing to C9orf72 regulation and C9orf72 loss of function we sought to characterize the transcriptional landscape of the C9orf72 locus taking a broader approach. By surveying the global CAGEseq expression data generated by single-molecule cDNA sequencing in the context of the FANTOM5 project [28] we observed that transcription at the C9orf72 locus has a complex architecture.

We found that the TSSs for the annotated C9orf72 transcripts are remarkably differentially expressed across samples, particularly between a subset of myeloid cells and CNS tissues. We detected novel non-annotated TSSs on the sense and antisense strand at the C9orf72 locus suggesting new potential transcripts and we observed changes in the expression of the annotated and newly identified C9orf72 transcripts, not only in C9orf72-HRE patients, but also in FTD patients carrying mutations in the microtubule associated protein tau $(M A P T)$ and granulin $(G R N)$ genes. We demonstrate that the repeat expansion cannot fully explain the reduction in C9orf72 expression and that additional molecular mechanisms contribute to the regulation of C9orf72 expression.

\section{Material and methods CAGEseq datasets}

Dataset 1: CAGEseq data published in the context of the FANTOM5 promoterome project [28], which generated a comprehensive map of TSSs and their usage across 975 human and 399 mouse samples (the samples used in this work to generate the individual and combined TSSs expression profiles of CNS and myeloid cells are listed in Additional file 1: Table S1). Dataset 2: CAGEseq data from 144 human brain samples consisting of seven anatomical regions (occipital-, frontal-, temporal lobe, hippocampus, caudate, putamen and cerebellum) from seven controls and three groups of familial FTD patients carrying a C9orf72-HRE, MAPT or GRN pathogenic mutation (list of samples provided in Additional file 1: Table S2). Dataset 3: CAGEseq data from human frontal lobe of 119 control donors with ages ranging from 2 to 95 years presenting no neurological symptoms at time of death (C. Blauwendraat, under review in another journal).

\section{Definition of distinct tag clusters at the human C9orf72 locus using FANTOM5 CAGEseq data}

From the ZENBU genome browser [29] we downloaded the coordinates of the decomposition-based peak identification (DPI) clusters identified by the FANTOM5 project in the genomic region chr9:27572518-27574055 (human genome build GRCh37/hg19), which includes the $5^{\prime}$ end of the C9orf72 gene and encompasses all the CAGEseq expression signals for the C9orf72 locus. Each DPI cluster, composed by multiple adjacent CAGEseq reads identifies a TSS. A detailed description of the DPI clustering method can be found in the supplementary information of [28] section 4, along with the definition of robust and permissive DPIs.

Fifteen TSSs have been identified in the region, four on the forward and 11 on the reverse strand (Additional file 1: Figure S1A-B). This original list of TSSs was adjusted based on the actual CAGEseq expression profiles 
(Additional file 1: Figure S1A) and resulted in the definition of a final set of nine TSSs, three on the forward and six on the reverse strand (Additional file 1: Figure S1C). TSSs on the reverse strand (same transcriptional direction as C9orf72) were named consistently with C9orf72 transcript variants when possible, with letters to separate distinct TSSs associated to the same variant (S4 indicates a putative novel transcript). TSSs on the forward strand were named AS (to reflect the fact that they are antisense to $C 9$ orf72) and numbered in the $5^{\prime}$ to $3^{\prime}$ direction.

Manual modifications to the original list of TSSs (Additional file 1: Figure S1D): we merged neighboring DPI clusters with similar expression profiles (giving rise to S4, AS1 and S2C TSSs), we extended two DPI clusters in order to capture all the CAGEseq signals corresponding to them (giving rise to S1 + S3a and AS3 TSSs) and we added two DPI clusters suggested by the permissive set of DPI clusters (TSSs AS2 and S1 + S3b). The remaining TSSs (S2B and S2A) were simply renamed and maintain the same coordinates as the original DPI clusters.

\section{Expression of C9orf72 TSSs as defined by CAGEseq using dataset 1}

For each of the TSSs defined as described above, we downloaded from ZENBU tag-count per million (tpm) normalized expression values for all the samples available in the FANTOM5 promoterome study. The downloaded expression values for CNS and myeloid cells are provided in Additional file 1: Table S1.

\section{Expression of C9orf72 TSSs as defined by CAGEseq using dataset 2}

CAGEseq libraries were prepared using a published protocol [30]. Briefly, total RNA from 144 brain samples (Additional file 1: Table S2) was used as starting material and sequenced on a HiSeq 2000 (Illumina). Sequenced reads were demultiplexed, trimmed using the FASTX toolkit (FASTX toolkit, http://hannonlab.cshl.edu/fas tx_toolkit/), filtered for artifacts using TagDust (version 1.12) [31] and mapped to the human genome (build GRCh37/hg19) using the Burrows-Wheeler Aligner (BWA version 0.5.9) [32] allowing up to two mismatches. The resulting BAM files were uploaded in ZENBU and the tpm normalized expression values of the C9orf72 TSSs were downloaded (available in Additional file 1: Table S2).

\section{Expression of C9orf72 TSSs as defined by CAGEseq using dataset 3}

Library preparation and creation of the BAM files containing mapped reads was performed as described above. The mapped CAGEseq reads were grouped into single base pair promoters by determining all positions in the genome to which the $5^{\prime}$ end of at least one CAGE read with mapping quality of at least 20 mapped to and outputting tpm normalized expression values per base pair position [33].

\section{C9orf72 expression in aging brain}

We analyzed the CAGEseq dataset 3 obtained from 119 frontal cortex brain samples to assess whether brain C9orf72 expression is influenced by age.

Pearson correlation calculations were made between each newly defined C9orf72 TSS and age value. To identify possible hidden confounding effects we also determined whether any of the C9orf72 TSS was influenced by RNA integrity number (RIN), gender and postmortem interval (pmi).

Weighted Gene Coexpression Network Analysis (WGCNA) We downloaded from the FANTOM5 data repository the BAM files corresponding to eosinophils, neutrophils, CD14+ monocytes and all adult CNS samples (the full list of samples included in the analysis is provided in Additional file 1: Table S1). After removing reads mapping to chromosomes $\mathrm{M}$ and $\mathrm{Y}$ from the downloaded BAM files, we used Python scripts designed at the RIKEN Omics Science Center [33] to group the remaining CAGEseq reads into TSSs and obtain the corresponding tpm normalized expression values. Because the major aim of the analysis was to infer information about $C 9$ orf72 function, we decided to include in the WGCNA only TSSs mapping to annotated genes. The data were filtered to ensure that all TSSs had expression above $1 \mathrm{tpm}$ in at least one sample and log-transformed, resulting in a dataset including 26,305 TSSs mapping to 17,267 distinct genes. Co-expression network construction, (including module membership calculation) was performed as previously described [34] and resulted in 18 co-expression modules, three of which contained all the TSSs mapping to C9orf72 (turquoise, yellow, blue). Module membership measure for each C9orf72 TSSs was also calculated. Of these only the S1+S3b TSS showed higher membership with respect to a different module (pink module). For the modules turquoise, yellow, blue and pink we calculated correlation values between the expression of C9orf72 TSSs and all the other TSSs assigned to the module. The correlations were calculated using the $\mathrm{R}$ function cor.test() with "spearman" method option, resulting in an association test based on Spearman's rho statistic. Adjusted $p$-values were calculated using the $\mathrm{R}$ function p.adjust(), with method = "BH" option (BenjaminiHochberg adjustment designed to control FDR). Networks and functional analysis were generated through the use of Qiagen's Ingenuity Pathways Analysis (IPA, QIAGEN Redwood City). 


\section{Donor samples used in quantitative PCR (qPCR) experiments}

Frozen tissue from disease and control donors was obtained from the Netherlands Brain Bank for the following brain regions: medial frontal and temporal gyrus, superior occipital gyrus, cerebellum, hippocampus, caudate and putamen. We obtained tissues from sporadic-FTD patients and patients with C9orf72-HRE, MAPT and GRN mutations, sporadic cases with Alzheimer's disease (AD), Progressive Supranuclear Palsy (PSP), ALS, Multiple Sclerosis (MS), Parkinson's disease (PD), cases with Huntington's disease (HD) and non-demented control donors.

Peripheral blood was collected from healthy donors and patients with clinical diagnosis of non familiar FTD, ALS and PD. Following appropriate informed consent genomic DNA, RNA and CD14+ monocytes were isolated for further analysis (see below). Details about brain samples are listed in Table 1 and details on blood samples are provided in Table 2.

All samples used in this study were screened for the presence of the C9orf72-HRE by primed repeat PCR according to established protocols [1, 2]. Reported mutations in MAPT and GRN mutations carriers were sequence verified. Brain donors with the diagnosis of sporadic FTD were also screened for mutations in the $M A P T$ and GRN genes. Additionally we amplified $1.6 \mathrm{~kb}$ in the C9orf72 promoter region in MAPT and GRN mutations carriers. PCR products were purified with EXOSAP (GE Healthcare) and sequenced by using BigDye terminators v3 on an ABI3500 system (Thermo Fisher Scientific). Primers sequences are supplied in Additional file 1: Table S3.

\section{DNA and RNA isolation}

Genomic DNA was isolated from peripheral blood and frozen brain tissue by using the DNeasy blood and tissue kit (Qiagen) following the manufacturer protocol.

RNA was isolated from frozen brain tissue, CD14+ monocytes and human microglia cells derived from fetal brain tissue purchased from 3H Biomedical with TRIzol reagent (Thermo Fisher Scientific) followed by DNAse treatment and further purification with the RNeasy columns (Qiagen). RIN was determined on a Bioanalyzer 2100 system (Agilent Technologies Inc.) (Table 1).

\section{CD14+ monocytes isolation}

CD14+ monocytes were isolated from peripheral blood mononuclear cells by density gradient using Ficoll-Paque ${ }^{\text {тм }}$ (GE Healthcare), followed by labeling with CD14 microbeads and magnetic separation with LS columns using the protocol provided by Miltenyi Biotech. CD14+ monocytes isolation with $>90 \%$ purity was verified by cell analysis on a FACSCalibur cytometer (BD Biosciences) using CellQuest software with gating on a live cell forward/side scatter gate, using the CD14 PE-conjugated and Mouse IgG2a-PE conjugated isotope control antibodies (Miltenyi Biotec) according to the protocol provided by the manufacturer.

\section{CDNA synthesis, strand specific reverse transcriptase PCR, 3'RACE and qPCR}

Total RNA primed with oligo dT (Qiagen) and random decamers (Thermo Fisher Scientific) was used for cDNA synthesis with Superscript III reverse transcriptase (RT) (Thermo Fisher Scientific) according to manufacturer's

Table 1 Characteristics of investigated brain samples

\begin{tabular}{|c|c|c|c|c|c|c|c|}
\hline \multirow{2}{*}{$\begin{array}{l}\text { Disease } \\
\text { code }\end{array}$} & \multirow{2}{*}{$\begin{array}{l}\text { Sample } \\
\text { number }\end{array}$} & \multirow[t]{2}{*}{ Age } & \multirow{2}{*}{$\begin{array}{l}\text { Female } \\
\text { frequency }\end{array}$} & \multicolumn{2}{|c|}{ Risk haplotype } & \multirow[t]{2}{*}{ pmi } & \multirow[t]{2}{*}{ RIN } \\
\hline & & & & Hom & Het & & \\
\hline NDD & 9 & $80.3 \pm 10.7$ & $4(50 \%)$ & 1 & 4 & $5: 46 \pm 1: 27$ & $8.4 \pm 0,8$ \\
\hline C9orf72-HRE & 10 & $64.9 \pm 9.0$ & $7(70 . \%)$ & 1 & 9 & $6: 18 \pm 1: 25$ & $7.4 \pm 1.2$ \\
\hline FTD-MAPT & 16 & $62.13 \pm 8.05$ & $8(50 \%)$ & 2 & 7 & $6: 11 \pm 1: 50$ & $7 \pm 1.1$ \\
\hline FTD-GRN & 8 & $61.6 \pm 8.1$ & $5(62.5 \%)$ & 0 & 2 & $4: 49 \pm 1: 00$ & $6.9 \pm 0,9$ \\
\hline$A D$ & 5 & $82 \pm 5.04$ & $3(60 \%)$ & 0 & 1 & $4: 31 \pm 1: 18$ & $7.2 \pm 1,5$ \\
\hline PSP & 5 & $71.6 \pm 9$ & $3(60 \%)$ & 0 & 5 & $6: 47 \pm 1: 46$ & $7 \pm 1.3$ \\
\hline FTD sporadic & 4 & $60.3 \pm 10.8$ & $2(50 \%)$ & 1 & NP & $6: 02 \pm 1: 18$ & $7.3 \pm 1.5$ \\
\hline ALS & 3 & $57,3 \pm 21.1$ & 1 (33.3 \%) & 0 & 1 & $5: 28 \pm 0: 27$ & $9 \pm 0.05$ \\
\hline $\mathrm{HD}$ & 3 & $53.3 \pm 6.6$ & 1 (33.3 \%) & 0 & 2 & $7: 16 \pm 2: 43$ & $4.9 \pm 0.6$ \\
\hline MS & 3 & $64.3 \pm 21.5$ & 2 (66.7 \%) & 0 & 2 & $6: 06 \pm 2: 48$ & $7.9 \pm 1.3$ \\
\hline PD & 3 & $79.7 \pm 8$ & 0 & 0 & 1 & $4: 55 \pm 1: 07$ & $7.9 \pm 0.9$ \\
\hline Total & 69 & & & $5(7.24 \%)$ & 34 (49.3 \%) & & \\
\hline
\end{tabular}

The table describes characteristics of brain samples used in this study: age, gender, sharing of risk haplotype, post mortem interval (pmi) and RNA integrity number (RIN). NDD non-demented donors, Age, pmi and RIN: per disease group indicated average and standard deviation

FTD-MAPT cases carrying pathogenic mutations: ten cases carrying the P301L mutation; four cases with the G272V mutation; one case with the R406W mutation and one case with the L315R mutation. FTD-GRN cases carrying pathogenic mutations: five cases carrying the S82Vfs mutation; one case with G300X mutation; one case with the C105fs mutation and one case carrying the Q24X mutation 
Table 2 Characteristics of the investigated CD14+ donor samples

\begin{tabular}{|c|c|c|c|c|c|c|}
\hline \multirow{2}{*}{$\begin{array}{l}\text { Disease } \\
\text { code }\end{array}$} & \multirow{2}{*}{$\begin{array}{l}\text { Sample } \\
\text { number }\end{array}$} & \multirow[t]{2}{*}{ Age } & \multirow{2}{*}{$\begin{array}{l}\text { Age at } \\
\text { onset }\end{array}$} & \multirow{2}{*}{$\begin{array}{l}\text { Female } \\
\text { frequency }\end{array}$} & \multicolumn{2}{|c|}{ Risk haplotype } \\
\hline & & & & & Hom & Het \\
\hline Controls & 5 & $35.2 \pm 13.3$ & NA & $40 \%$ & NT & NT \\
\hline C9orf72-HRE & 4 & $62.5 \pm 9.9$ & $58 \pm 12.3$ & $50 \%$ & 2 & 2 \\
\hline ALS & 9 & $65.9 \pm 6.8$ & $62.8 \pm 6.5$ & $44.4 \%$ & 0 & 1 \\
\hline FTD & 9 & $63 \pm 13.2$ & $60.3 \pm 13.3$ & $33.3 \%$ & 0 & 3 \\
\hline PD & 10 & $66.8 \pm 9.4$ & $56.5 \pm 7.9$ & $30 \%$ & 0 & 5 \\
\hline
\end{tabular}

The table summarizes characteristics (age, age at onset, gender, sharing of risk haplotype) of CD14+ sample donors NA not applicable, NT non-tested. Age and age at onset: per group indicated average and standard deviation

specifications. Possible DNA genomic contamination was excluded by adding $\mathrm{RT}(-)$ reactions.

Strand specific RT- PCR (ssRT-PCR) was performed according to Chung et al [35].

RACE ready mRNA for 3'RACE experiments were performed using the SMARTer RACE cDNA amplification kit (Clontech Laboratories) according to the manufacturer protocol. Primers sequences are given in Additional file 1: Table S3.

qPCR was carried out in triplicate on a ViiA7 real time PCR system (Thermo Fisher Scientific) using SYBR Green PCR master mix (Thermo Fisher Scientific) and 0,04 $\mu \mathrm{M}$ specific primer pairs for all targets. For a subset of samples we performed qPCR with Taqman assays for total C9orf72 and transcript 3 in triplicate using Taqman Gene expression Master mix (Thermo Fisher Scientific) according to the procedure recommended by the manufacturer.

Normalized relative quantities (NRQ) were calculated using qbase ${ }^{+}$version 2.4 (Biogazelle) with $B 2 M, H M B S$, RPLPO and OAZ1 as reference targets [36]. The geNorm module in qbase $^{+}$was used to compute expression stability values for all reference targets and determine the optimal number of reference targets for every experiment. Variables were compared using nonparametric Mann-Whitney tests and a $p$-value of 0.05 was considered significant.

To measure absolute C9orf72 quantity in brain and monocytes cDNAs, we performed digital PCR using the Quant studio 3D system (Life Technologies) using cDNA serial dilution (25 ng-3.125 ng RNA range) and Taqman assays for C9orf72 total and transcript 3 according to the manufacturer 's protocol.

Primers for C9orf72 total, transcript 1,2 and 3 were obtained from Waite et al. [37]. Primer sequences for S4-TSS, AS1 and AS3 are listed on Additional file 1: Table S3.

\section{C9orf72 expression quantitative trait loci analysis using CAGEseq expression values}

Genome wide genotypes data for 119 frontal lobe samples for which we have CAGEseq expression values were obtained as described in Blauwendraat et al (C. Blauwendraat under review in another journal).
We selected 20 Single Nucleotide Polymorphisms (SNPs) defining the C9orf72-HRE risk haplotypes as described by Mok et al [38] and haplotypes were constructed based on the reported risk allele allowing maximum two mismatches per individual. Expression quantitative trait loci (eQTL) analysis was performed using MatrixeQTL [39] testing the expression level of the newly defined TSSs with the frequency of risk haplotypes per individual, using as covariates pmi, age, gender and RIN.

\section{C9orf72 eQTL analysis using normalized relative quantities expression values}

Normalized relative quantities (NRQ) expression values were generated in qPCR experiments for the different C9orf72 targets as described above.

Five SNPs were selected for genotyping based on the reported risk allele and their genomic location: rs10757665, rs10757668, rs3849942, rs2453556, and rs702231. Haplotypes were constructed to identify carriers of the C9orf72$H R E$ risk allele allowing maximum two mismatches per individual at the ends of the haplotype as previously described [38]. eQTL analysis was performed using MatrixeQTL correlating the NRQ expression levels of the qPCR targets with the frequency of risk haplotypes per individual using as covariates brain region, disease status and disease mutation.

\section{C9orf72 promoter methylation assay}

Quantitative assessment of methylation levels was determined by using a methylation-sensitive restriction enzyme DNA digestion coupled with qPCR [40].

We applied Mann-Whitney U tests to compare methylation levels between C9orf72-HRE patients and controls, MAPT mutation carriers and controls and GRN mutations carriers and controls. Pearson correlation analysis was performed to correlate methylation values with risk haplotype frequency and expression.

\section{Knock down experiments}

Knock down experiments were performed on BE(2)M17 cell lines (ATCC ${ }^{\oplus}$ CRL-2267 $^{\mathrm{TM}}$ ) using short hairpin RNA (shRNA) plasmids for C9orf72 transcripts, 2 and 3, 
MAPT, GRN and scrambled control (Sigma, TRC 1 and $1.5)$ as described in [41].

\section{RNA FISH and analysis of RNA foci burden}

CD14+ monocytes from three C9orf72-HRE patients and three FTD cases HRE negative were seeded onto Poly-L-lysine-coated glass coverslips by cytospin at $120 \mathrm{~g}$ for $5 \mathrm{~min}$, fixed in $4 \%$ paraformaldehyde and stained with 5' TYE563-labelled $[\mathrm{CCCGG}]_{3}$ probe (sense foci), or [GGGGCC] $]_{3}$ probe (antisense foci) (Exiqon) as previously described [5]. Nuclei were stained with Hoechst and slides were subsequently imaged on a Leica TCS SP8 confocal microscope using a Leica Plan Apochromat $100 \times$ oil immersion objective lens. The resulting images were analyzed using an internally developed pipeline in CellProfiler [42]. Briefly, maximum projections of each image channel were made in Fiji [43] and used as input in the pipeline for at least 70 cells for each patient and probe. Threshold value was automatically set for each image and nuclei were detected. Speckles were then enhanced in the RNA foci channel, and this channel was subsequently masked based on the nuclei channel. Threshold was set per object basis using the Mixture of Gaussian algorithm. The RNA foci were then automatically counted and assigned to a nucleus, and the percentage of nuclei containing at least one focus was determined for each sample.

\section{Statistical analysis and plots}

All statistical analyses, correlations and plots were performed using the free software environment $\mathrm{R}$ (Environment R, (https://www.r-project.org/).

\section{Ethical approval and consent to participate}

Frozen tissues used to generate the CAGEseq dataset 2 and used in qPCR studies was obtained from the Netherlands Brain Bank (NBB). All tissue requests received at the NBB are reviewed by NBB's scientific committee and all material and data collected by the NBB are obtained on the basis of written informed consent. Procedures, information and consent forms of the NBB have been approved by the Medical Ethics Committee of the VU Medical Centre. The use of human brain samples for the work leading to the generation of the CAGEseq dataset 3 was approved by the NIH Office for Human Subjects Research as indicated in C. Blauwendraat, under review in another journal. Control and disease blood donors gave informed consent and study approval was obtained by the Ethics Committee of the University of Tübingen.

\section{Results}

The C9orf72 locus shows a complex transcriptional architecture in the CNS and in CD14+ monocytes

To characterize the transcriptional events occurring at the C9orf72 locus and identify possible new transcripts
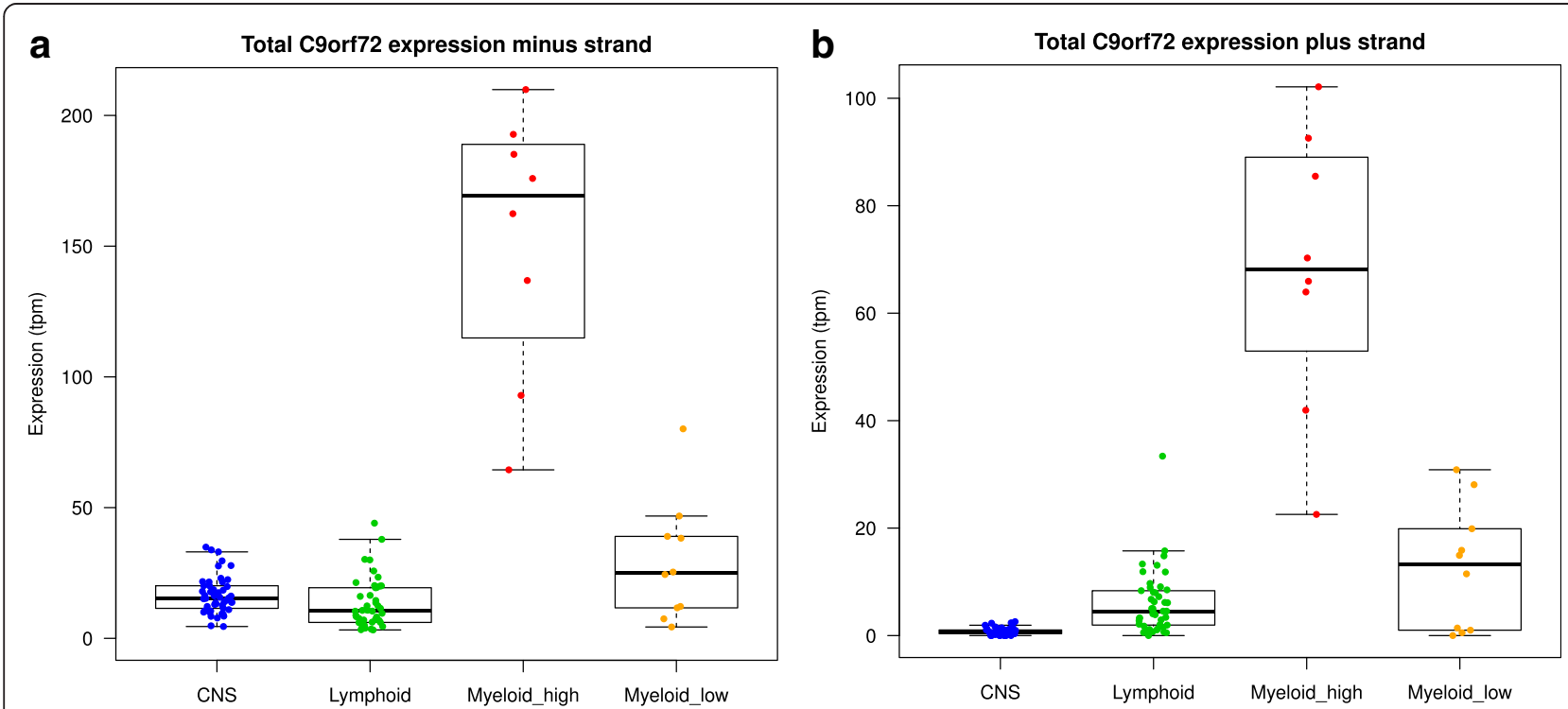

Fig. 1 Global analysis of C9orf72 expression using CAGEseq expression data from the FANTOM5 project. Boxplots showing total C9orf72 expression at the C9orf72 locus from the sense (a) and antisense (b) strand in myeloid, lymphoid and CNS samples available in the FANTOM5 promoterome sample collection. C9orf72 expression is particularly high in a subset of myeloid cells (CD14+ monocytes, eosinophils and neutrophils) indicated as myeloid-high and lower in macrophages and mast cells (myeloid-low), T and B cells (lymphoid cells) and tissues from the CNS. Expression values are indicated on the Y-axes as tpm. Total expression at C9orf72 locus deriving from the sense strand (reverse or -) was calculated by adding the expression of all the TSSs on the reverse strand (i.e. S1 + S3b, S1 + S3a, S2A, S2B, S2C and S4) for all the libraries included in the study. Similarly total expression deriving from the antisense strand (forward or + ) was calculated adding the expression of all the TSSs located on the forward strand (i.e. AS1, AS2 and AS3) 
on the sense and antisense strands that contribute to C9orf72 expression, we used publically available CAGEseq expression data generated in the context of the FANTOM5 project [28] to investigate in which tissues and cells C9orf72 is expressed. We observed C9orf72 was expressed above $1 \mathrm{tpm}$ in the sense strand in $83.3 \%$ of the samples and in the antisense strand in $22.1 \%$ of the samples. Particularly, C9orf72 expression was very high in CD14+ monocytes, eosinophils, and neutrophils, a subset of myeloid cells involved in innate and adaptive immunity, from now on referred to as myeloid-high (Fig. 1a and b). C9orf72 expression levels in other myeloid cells such as mast cells and macrophages that are derived from monocytes (further referred to as myeloid-low), or lymphoid-derived cells like $\mathrm{T}$ and $\mathrm{B}$ cells were much lower (Fig. 1a and b). In the remaining cell types and tissue samples throughout the body including the CNS, C9orf72 expression levels were generally low and comparable to lymphoid cells.

Interestingly we observed an increase in C9orf72 expression from both strands after challenging CD14+ monocytes with a range of pathogens including Candida, Cryptococcus, Streptococcus group A and Salmonella that induce an immune response (Additional file 1: Figure S2). Taken together these findings suggest C9orf72 might play a role related to myeloid function in immune response and the maturation of monocytes into macrophages.

We then analyzed TSSs expression profiles at the C9orf72 locus, from the myeloid-high and CNS samples, in more detail. Three coding transcripts are currently annotated for the C9orf72 gene: NM_145005 (Transcript 1), NM_18325 (Transcript 2) and NM_1256054 (Transcript 3) (Fig. 2a). The C9orf72-HRE is located in intron 1 considering transcripts 1 and 3 , or at the promoter region considering transcript 2 . Our combined TSSs profile (Fig. $2 \mathrm{~b}$ ) shows that transcripts 1 and 3 share two TSSs referred to as $\mathrm{S} 1+\mathrm{S} 3 \mathrm{a}$ and $\mathrm{S} 1+\mathrm{S} 3 \mathrm{~b}$. The $\mathrm{S} 1+\mathrm{S} 3 \mathrm{a}$ TSS is mainly expressed in myeloid-high cells, while the $\mathrm{S} 1+\mathrm{S} 3 \mathrm{~b}$ TSS is mainly used in the CNS (Fig. 2b and Additional file 1: Figure S3). Transcript 2 uses three TSSs: S2A, S2B and S2C. The S2A TSS is expressed at comparable levels in CNS and myeloid cells (similar average expression as indicated in Additional file 1: Figure S3), while S2B and S2C TSSs are mainly expressed in myeloid cells (Fig. 2b and Additional file 1: Figure S3).

We observed additional TSSs, expressed mainly in myeloid cells that indicate potential novel transcripts at the C9orf72 locus: a TSS on the reverse strand downstream of exons $1 \mathrm{~A}$ and $1 \mathrm{~B}$ of the annotated transcripts (S4 TSS in Fig. 2b) and three TSSs on the forward strand (AS1, AS2 and AS3 TSSs in Fig. 2b). By performing a series of RT-PCR and ssRT-PCR experiments in combination with 3' RACE from RNA of CD14+ monocytes we confirmed the existence of five new transcripts at the C9orf72 locus in C9orf72-HRE patients and controls, two additional C9orf72 transcripts and three transcripts antisense to C9orf72 (Fig. 2c and Additional file 1: Figure S4). The novel C9orf72 sense transcripts differ from the annotated ones only by an alternative exon 1 downstream the HRE (Fig. 2c), 265 bp long with a conserved donor splice site (AAG/gtacgt). Usage of the alternative exon 1 does not yield additional translational start sites and therefore does not change the protein composition. The three antisense C9orf72 transcripts AS1, AS2 and AS3 (Fig. 2c) are unspliced. CAGEseq data and 3'RACE experiments show that AS1, located in intron 1b starts 657 base pairs upstream of the HRE but it does not encompass the HRE region. While we cannot rule out a technical artifact in our experimental set up because of the difficulty to reverse transcribe the HRE region, our findings are in agreement with a previous study that showed antisense transcripts starting in intron 1b, (251-455 bp upstream the repeat) but not extending to the sense exon 1a region adjacent the HRE [12]. The C9orf72-AS2 and -AS3 transcripts overlap for $606 \mathrm{bp}$ and they are located head-to-head to C9orf72 downstream the HRE (Fig. 2c).

The distinct modes of expression of C9orf72 TSSs in $\mathrm{CNS}$ and myeloid cells are mirrored at a functional level based on the WGCNA performed on CNS, monocytes, eosinophils and neutrophils samples (Additional file 1: Table S4, and Additional file 1). Our functional enrichment analysis showed that the S2A expression profile correlates with genes involved in brain-related biological processes like synaptic transmission and vesicle transport, while the TSSs that are mainly expressed in myeloid cells are associated with immune response (Additional file 1: Table S5). Interestingly, the S1 + S3b TSS for transcript 1 and 3 is significantly correlated (rho $=0.49$, adjusted $p$-value $=0$,) with the IPO7 gene encoding for importin 7, a nucleocytoplasmic transport protein [44] when considering correlations in the pink module, with which the S1 + S3b TSS showed highest membership (Additional file 1).

\section{C9orf72 expression levels in CNS do not change during development and aging}

Since human FANTOM5 data showed considerable differences in C9orf72 expression between myeloid cells and CNS, we investigated whether this finding was also true across distinct CNS regions. In addition to the FANTOM5 collection that contains data for a range of CNS regions in a limited numbers of adult donors, we analyzed an additional CAGEseq brain dataset consisting of 49 control libraries generated in our laboratory, with data from seven CNS regions from seven control donors (Additional file 1: Table S2). In both datasets all the TSSs for the sense transcripts showed the highest expression in cerebellum, followed by regions belonging to the cortex-limbic group and striatum (Fig. 3 and Additional 


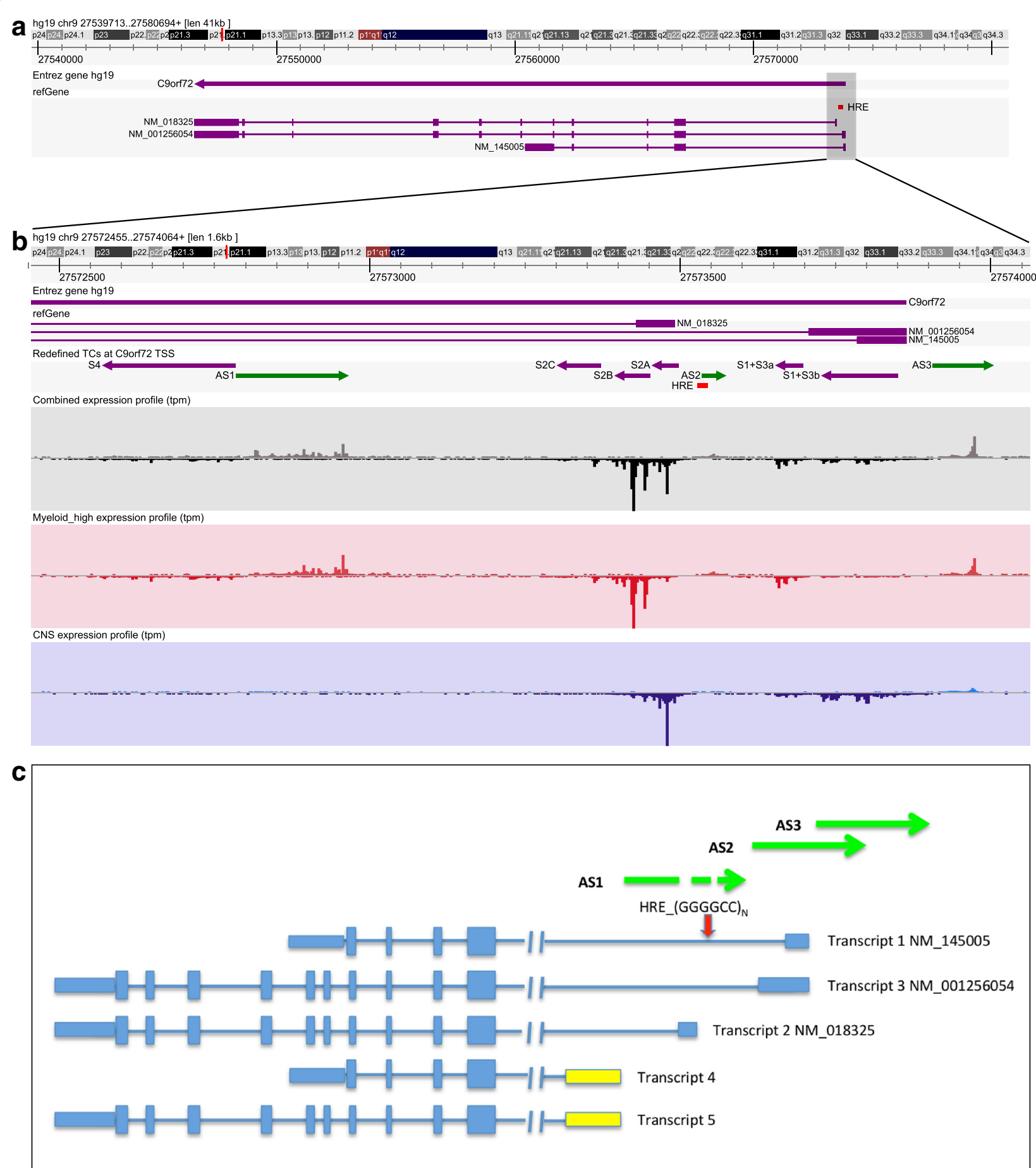

Fig. 2 (See legend on next page.) 
(See figure on previous page.)

Fig. 2 C9orf72 locus. a Represents the three coding transcripts for the C9orf72 gene located on the reverse strand of chromosome 9: NM_145005 (transcript 1), NM_18325 (transcript 2) and NM_1256054 (transcript 3). The HRE is located either in intron 1 considering transcripts 1 and 3, or at the promoter region considering transcript 2. The region captured in the figure encompasses the $41 \mathrm{~kb}$ identified by the hg19 coordinates chr9:27539713-27580694. b Zooming in of the 1,6 kb region identified by the hg19 coordinates chr9:27572455-27574064 (grey box in a) and depicting the $5^{\prime}$ end of the C9orf72 locus as represented by the ZENBU Genome Browser. As indicated by the labels in the left side of each track, the following features are depicted from top to bottom: Entrez gene; refGene transcripts; C9orf72 TSSs (the details about their definition is available in material and methods); the CAGEseq expression profile obtained combining myeloid, lymphoid and CNS samples available in the FANTOM5 tissue collection; the expression profile restricted to myeloid-high samples and the expression profile restricted to CNS samples. The height of the expression profiles is scaled to the maximum value for the corresponding set of samples and it clearly highlights the differences between TSS usage in CNS and myeloid-high samples. The actual expression levels are represented as tpm in Additional file 1: Figure S3. c Schematic representation of the C9orf72 locus showing the three AS transcripts C9orf72-AS1, C9orf72-AS2 and C9orf72-AS3, C9orf72 annotated transcripts 1,2 and 3 and the newly identified C9orf72 transcripts 4 and 5. The dash-arrow in C9orf72-AS1 indicates the transcript might be longer than what detected in our experimental set-up. C9orf72-AS is located at chr9: 27573532-27574512 (hg 19) immediately adjacent to the C9orf72-HRE. C9orf72-AS3 chr9: 27573906-27575066 (hg19) starts 40 bp downstream exon 1a of C9orf72 presents a conserved poly-adenylation (poly-A) 692 bp from the $5^{\prime}$ end and an alternative poly-A site 1029 bp from the $5^{\prime}$ end

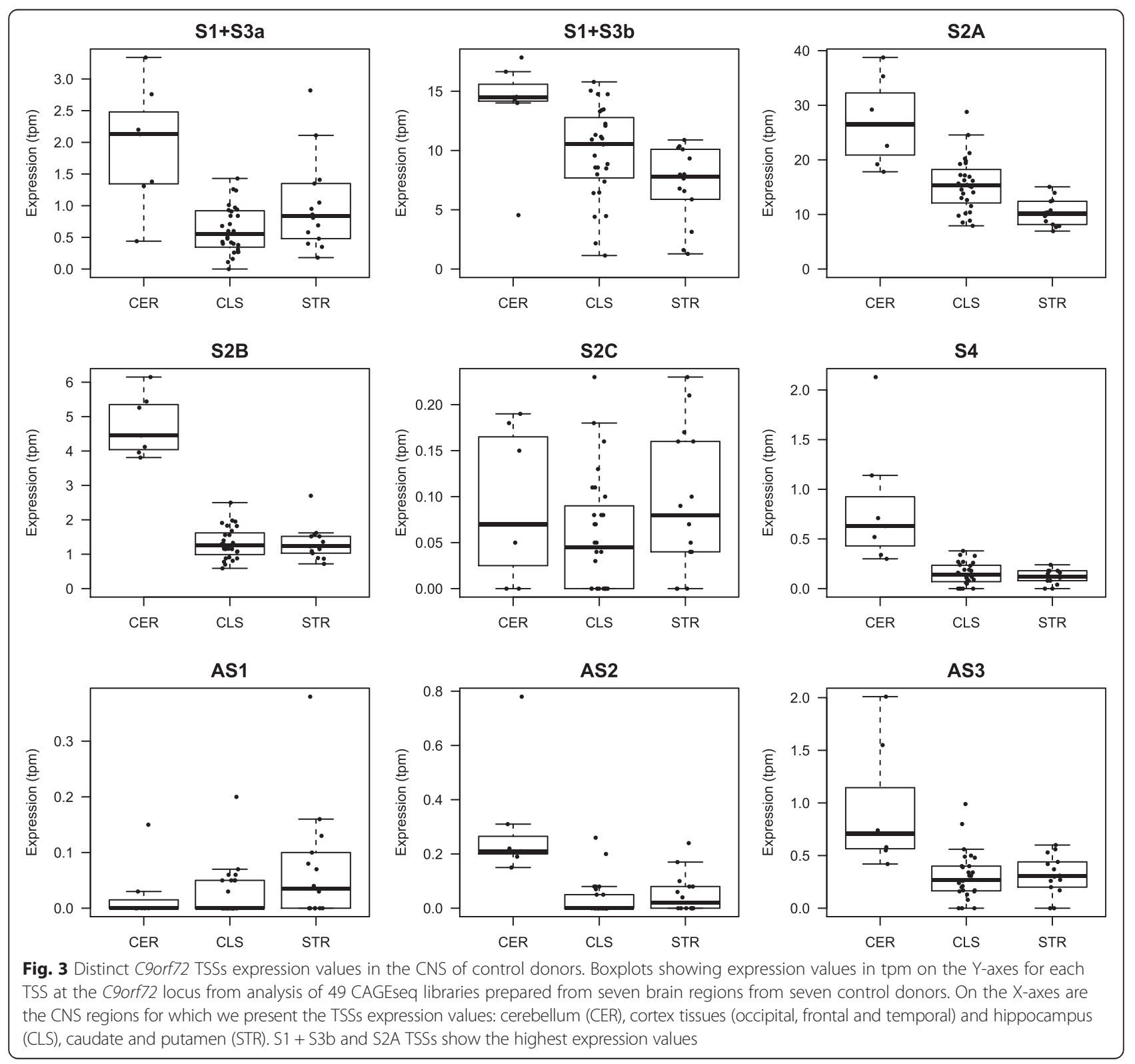


file 1: Figure S5). Expression levels of the TSSs for the antisense transcripts were generally low or absent in specific CNS regions. Only AS3 TSS displayed expression above zero in all the CNS regions.

We next investigated C9orf72 expression differences between fetal and adult cortex. The expression of C9orf72 TSSs was generally similar in fetal and adult samples (Additional file 1: Figure S6) suggesting C9orf72 expression levels do not significantly change during development and aging, which is in agreement with previous studies based on microarrays [45]. To confirm this result we analyzed an additional CAGEseq dataset of 119 frontal cortex brain samples with age range from 2 to 95 years from control donors generated in our laboratory. Pearson correlations between each newly defined C9orf72 TSS and age value showed no evidence that aging influences C9orf72 expression (data not shown).

The human FANTOM5 collection does not contain data on C9orf72 expression in microglia. Therefore we isolated RNA from commercially available microglia from human fetal brain tissue. Digital PCR experiments (Additional file 1: Figure S7) showed C9orf72 is lowly expressed in microglia as compared to CD14+ monocytes or total brain tissue. This finding would need however to be further investigated in adult microglia.

\section{C9orf72 expression levels are reduced in FTD brains}

It has been shown that the C9orf72-HRE generates truncated transcripts containing the repeat sequences [17, 46] therefore we wanted to determine whether the HRE sequence itself in addition harbors a TSS that could generate additional HRE-containing transcripts. CAGEseq is a technique particularly suitable for detecting TSSs of 5 '-capped transcripts generated by RNA polymerase II. Analysis of our CAGEseq libraries showed no evidence for the presence of a TSS within the repeat sequence suggesting that there is either no transcription starting within the repeat or, alternatively, there is a TSS within the repeat but the corresponding transcript that would contain the expanded repeat is not capped and therefore cannot be detected with CAGEseq.

Next we investigated whether the presence of the HRE would influence the expression levels of the surrounding TSSs at the C9orf72 locus in brain tissue of C9orf72HRE patients as compared to controls. Several reports have indeed observed a decrease of $C 90 r f 72$ expression in brains of C9orf72-HRE carriers albeit with some inconsistencies [1, 13, 19, 37, 46-50]; however these studies investigated the expression level for the three C9orf72 annotated transcripts only. We looked at our CAGEseq data obtained from brain tissue of FTD patients carrying the C9orf72 repeat expansion and controls. In all the investigated regions we observed a consistent reduction in expression for $\mathrm{S} 1+\mathrm{S} 3 \mathrm{a}, \mathrm{S} 1+\mathrm{S} 3 \mathrm{~b}$ and S2A TSSs in C9orf72-HRE cases as compared to control brains. In contrast we found a small increase in AS1 TSS in all brain regions and a small increase in AS3 TSS in frontal and hippocampus in cases compared to the controls (Fig. 4 and data not shown).

A recent study observed a $C 9$ orf 72 reduction in FTD patients without a C9orf72-HRE but this observation was not investigated in detail [19]. We therefore expanded our study to include FTD cases with $M A P T$ and GRN mutations without the HRE. In these cases we observed decreased expression for $\mathrm{S} 1+\mathrm{S} 3 \mathrm{a}, \mathrm{S} 1+\mathrm{S} 3 \mathrm{~b}$ and S2A TSSs in all the investigated areas. AS3 TSS showed to be slightly increased in frontal lobe and hippocampus, while no differences where observed in AS1 TSS expression (Fig. 4 and data not shown).

To confirm and extend these findings we investigated C9orf72 expression of all transcripts by qPCR experiments. We used a set of primers designed to target the annotated and the novel transcripts variants identified in this study in the sense and antisense orientation in a group of patients carrying C9orf72-HRE, MAPT and GRN mutations and control donors across several brain regions (see Table 1).

Consistent with our CAGEseq findings we observed reduction of all $C 90$ orf72 annotated transcripts in the C9orf72 patients as well as in the GRN and MAPT mutation carriers in particular in frontal and temporal cortex and hippocampus (Fig. 5a-b and data not shown).

Statistical analysis on data from the frontal lobe area, for which we had the largest number of samples available, showed that the observed C9orf 72 reduction was significant in all the cases with the only exception of transcript 2 in GRN and MAPT mutation carriers (Additional file 1: Table S6).

We then investigated whether and to which extent the newly detected C9orf72 transcripts were also differentially expressed in these FTD cases. In our experimental setup we could not reliably detect S4 TSS in brain samples (as it is mainly expressed in myeloid cells) and therefore we excluded it from further analysis. In agreement with our CAGEseq data we observed an increase for AS1 in the C9orf72-HRE patients and AS3 TSSs in the C9orf72-HRE and GRN patients as compared to controls (Fig. 5c). However only the increase for AS3 expression reached significance. Interestingly we observed a negative correlation between total C9orf72 expression and AS3 in C9orf72-HRE cases (Spearman correlation -0,871).

\section{Aspecific neurodegenerative processes do not explain C9orf72 reduction in FTD brains}

Our finding that $C 90 r f 72$ expression is also reduced in non-HRE FTD patients suggests that the reduction of C9orf72 expression is not entirely dependent on the 


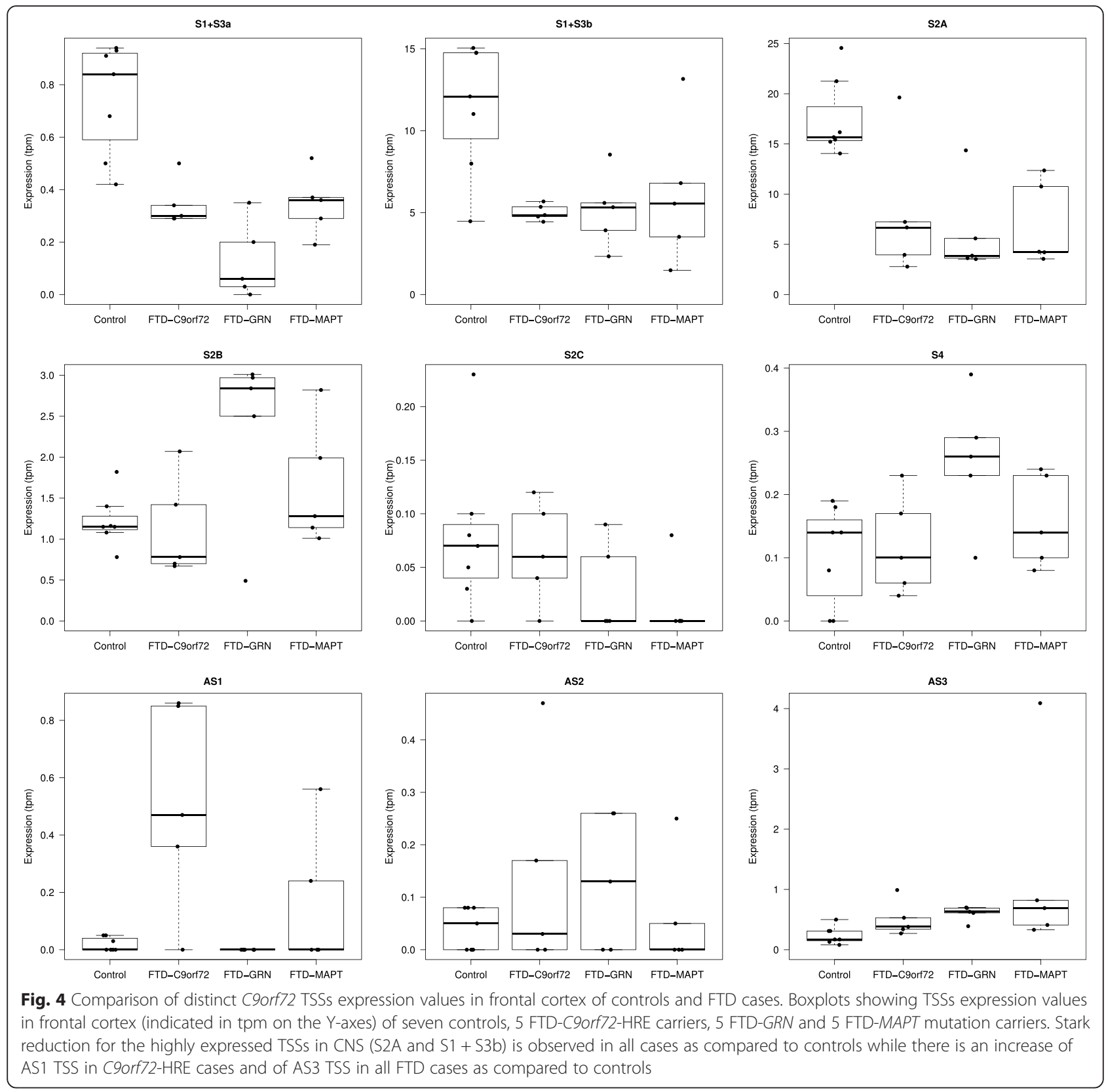

repeat expansion. Sequence analysis of the GC-rich lowcomplexity sequence (LCS) adjacent to the HRE did not reveal any DNA variants, including the 10 bp deletion observed in up to $25 \%$ of C9orf72-HRE patients [51, 52] that might explain the reduction in C9orf72 expression; therefore we explored other potential causes. One possible explanation might be that the observed C9orf 72 decrease in GRN and MAPT cases is a consequence of the neurodegeneration process. We therefore performed a series of qPCR experiments on cDNA from brain RNA of patients with different neurodegenerative disorders including sporadic FTD, AD, ALS, PSP, PD, HD and MS patients (Table 1). In the striatum in all cases other than
C9orf72-HRE, FTD-MAPT and FTD-GRN we did not observe a reduction in total C9orf72 expression as compared to control brains; in contrast a trend towards an increased expression could be observed in PD cases, although the finding needs to be replicated in more samples (Additional file 1: Figure S8A). In the frontal area, sporadic FTD and AD cases showed high variability in C9orf72 expression and no clear reduction of total C9orf72 as compared to control brains (Additional file 1: Figure $\mathrm{S} 8 \mathrm{~B})$. Our data therefore suggest that the C9orf72 reduction we observed in MAPT and GRN cases is not merely due to a non-specific neurodegenerative process. 


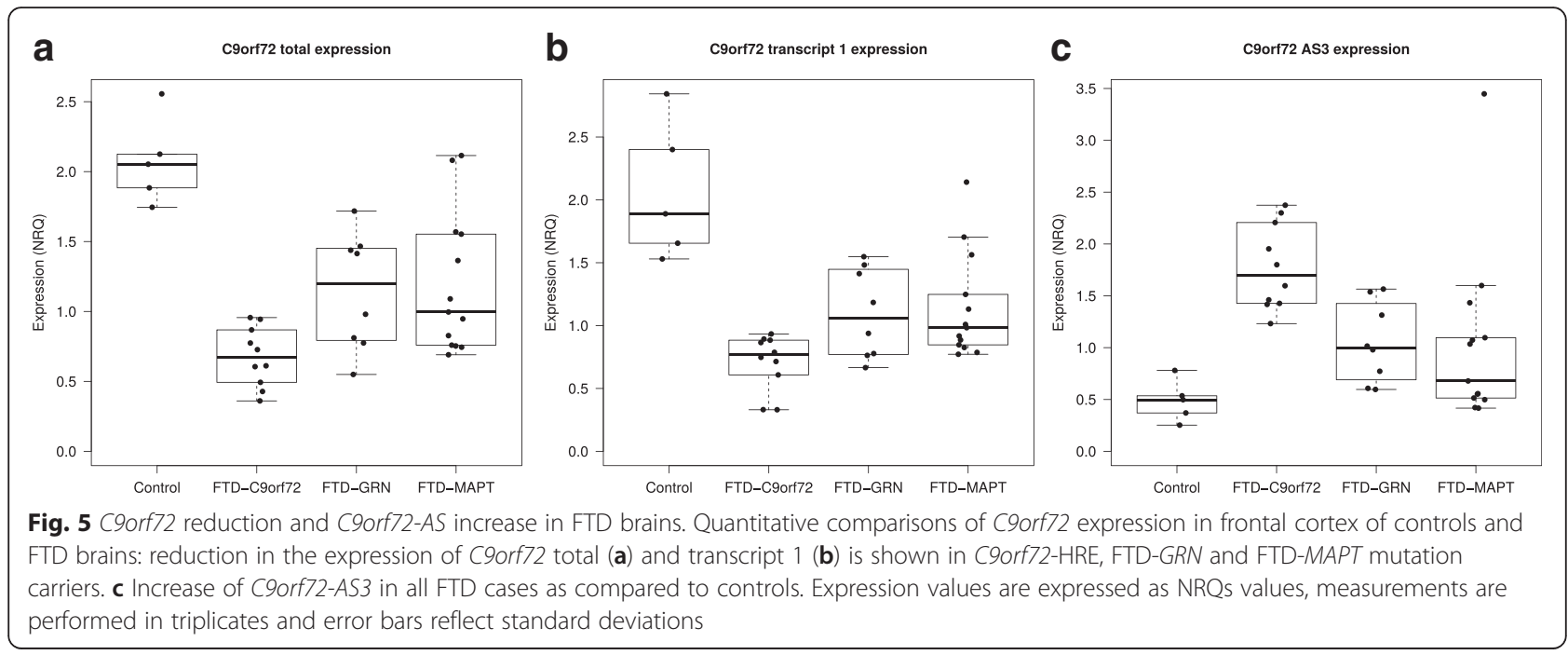

C9orf72-HRE risk haplotype influences C9orf72 expression It has been reported that the C9orf72-HRE exclusively occurs within a $110 \mathrm{~kb}$ risk haplotype [38] common in individuals of Northern European ancestry. We investigated if the observed C9orf72 reduction in non-HRE carriers could be explained by the risk haplotype. We first performed eQTL analysis on 119 frontal cortex control brain samples from which we have expression and genotype information. Based on the 20 SNP risk haplotype as defined by Mok et al [38], we identified seven individuals homozygous for the risk haplotype and 46 heterozygous individuals. The remaining 66 individuals were homozygous for the non-risk haplotype.

Our eQTL analysis shows that the risk haplotype is associated with higher expression of the C9orf72 TSSs for transcripts $1+3$ (FDR values $2.95 \mathrm{eE}-10$ and $4.25 \mathrm{E}-20$ for
$\mathrm{S} 1+3 \mathrm{a}$ and $\mathrm{S} 1+3 \mathrm{~b}$ TSSs, respectively) (Fig. 6a and b) and to lower expression of the C9orf72 TSSs for the most abundant transcript 2 (FDR values 0.010 and 0.013 for S2A and S2B TSSs, respectively) (data not shown). On average, we observed a three fold increase in the expression of S1 + 3a and S1 + S3b TSSs in the individuals homozygous for the risk haplotype as compared to the individuals carrying the non-risk haplotype and a 1.2 and 1.5 decrease in the expression of S2a and S2b TSSs, respectively.

Next we determined by genotyping which samples used in our qPCR experiments on brain samples were carrying the risk haplotype. We identified five individuals homozygous and 34 heterozygous for the risk haplotype and 29 individuals non-carriers of the risk haplotype (Table 1).
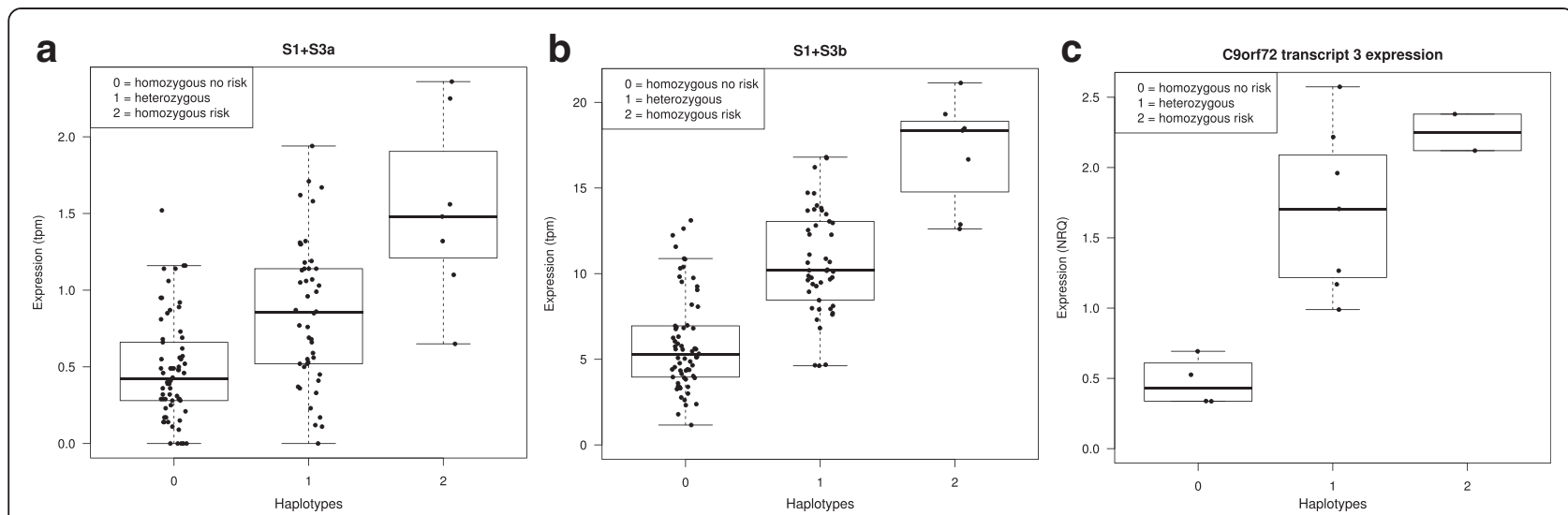

Fig. 6 Influence of C9orf72-HRE risk haplotype on C9orf72 expression. Boxplots showing the association between the C9orf72-HRE risk haplotype and expression in tpm for C9orf72 S1 + S3a (a) and S1 + S3b (b) TSSs. Individuals carrying the risk haplotype in heterozygous and homozygous state have a higher expression value as compared to carriers of the non-risk haplotype. Boxplots (c) show quantitative comparison of C9orf72 transcript 3 in FTD-MAPT non-carrying the risk haplotype, heterozygotes and homozygotes for the risk haplotype. Expression values are expressed as NRQs values 
We then correlated C9orf72 transcripts expression levels determined by qPCR with the concordance to the risk haplotype. In our $\mathrm{qPCR}$ experiments we could measure the effect of the risk haplotype C9orf72 transcript 1 and 3 in separate assays differently than with CAGEseq as C9orf72 transcript 1 and transcript 3 share the same TSS. We observed a significant association of the risk haplotype with a higher expression of C9orf72 transcript 3 in agreement with our CAGEseq eQTL findings (FDR value 0,0004 ) but not with transcript 1 , with the influence of the risk haplotype on C9orf72 transcript 3 clearly shown in Fig. 6c. We could not replicate the association of the risk haplotype with a decrease of C9orf72 transcript 2 probably because of the small effect and sample size.

Taken together these results suggest that the risk haplotype affects C9orf72 expression in brain tissue, but it does not fully explain the observed C9orf 72 reduction in MAPT and GRN patients as it was also detected in cases not carrying the risk allele. Therefore additional mechanisms must exist to explain the observed C9orf 72 decrease.

\section{C9orf72 promoter is not methylated in MAPT and GRN mutations carriers}

Several studies have shown that hypermethylation and $\mathrm{H} 3$ and $\mathrm{H} 4$ lysine trimethylation of the C9orf72 promoter region are associated with down-regulation of C9orf72 mRNA expression in HRE patients [26, 48, 53], with no hypermethylation observed in normal or intermediate expanded alleles (up to 43 repeats). However to the best of our knowledge, the methylation level of the C9orf72 promoter in FTD cases with MAPT and GRN mutations has not been yet investigated.
We therefore performed an HhaI methylation-sensitive digestion assay combined with $\mathrm{QPCR}$ as described by Russ and colleagues [40]. Among the C9orf72-HRE cases, two samples were strongly hypermethylated and overall C9orf72 promoter methylation levels were higher in C9orf72 patients as compared to controls, MAPT and GRN mutations carriers ( $p$-value 0.001644, p-Value 0.01977 and p-value 0.007349 Wilcoxon rank-sum test) while no significant differences were observed between $M A P T$ cases versus controls and GRN cases versus controls, ruling out a direct influence of C9orf 72 methylation level on C9orf72 expression in these patients. We then investigated whether the risk haplotype would influence the methylation level of the C9orf 72 promoter in the MAPT and GRN mutation carriers. We did not observe a significant association between the risk haplotype, in the homozygous or heterozygous state and C9orf72 promoter methylation level (data not shown). Our results therefore suggest that $C 9$ orf 72 promoter methylation level by itself or in combination with the risk haplotype does not account for the C9orf72 reduction observed in MAPT and GRN cases.

\section{Possible interaction within C9orf72, MAPT and GRN molecular pathways}

The C9orf72 reduction observed in MAPT and GRN mutation carriers could also imply a functional interaction within the molecular pathways disrupted by the C9orf72, $M A P T$ and GRN mutations respectively. In this respect the decrease in MAPT transcripts containing exon 10 observed by Prudencio et al in RNAseq data from C9orf72-HRE patients is interesting [9]. This observation could be explained by a general impairment in RNA processing as downstream consequence of the HRE [9].
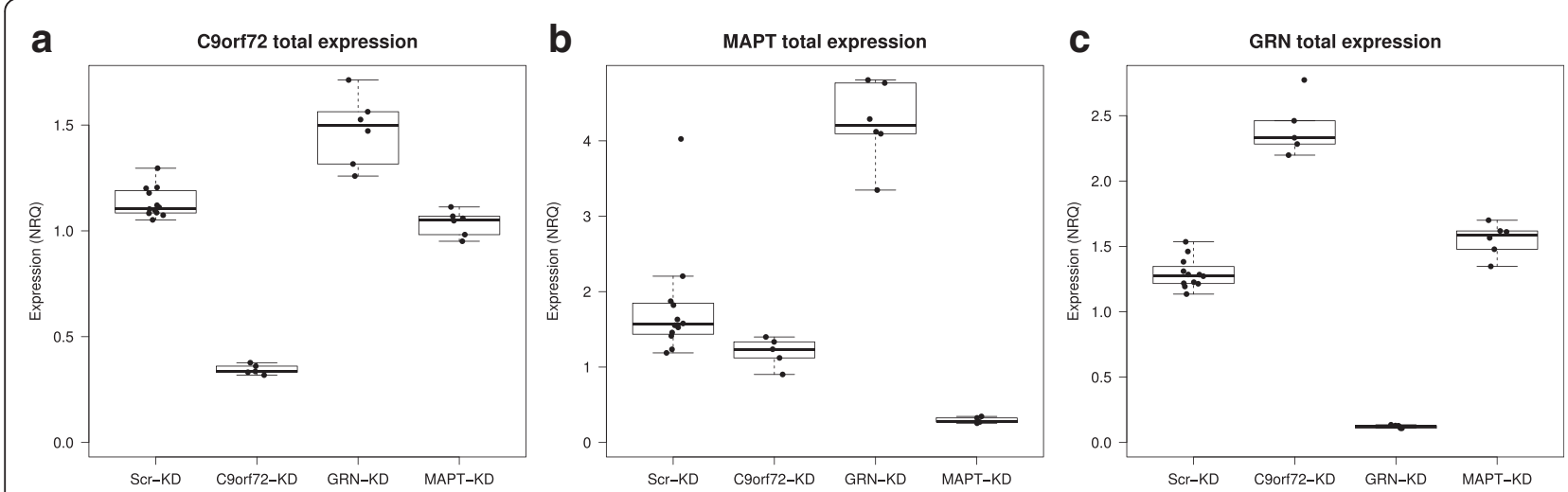

Fig. 7 Changes in C9orf72, MAPT and GRN expression in BE(2)M17 cells after knockdown of C9orf72, MAPT and GRN. Boxplots showing changes in total C9orf72, MAPT, GRN relative expression values in BE(2)M17 cells after knockdown C9orf72, MAPT, GRN and scrambled sequences in separate experiments for $\mathrm{N}=6$. Knockdown targets are indicated on the $\mathrm{X}$-axes and $\mathrm{NRQ}$ values are depicted on the $\mathrm{Y}$-axes. a Decrease C9orf72 relative expression can be observed after knockdown of MAPT, while silencing GRN results in an increase of C9orf72 expression. b Decrease in MAPT expression is observed after knockdown of C9orf72 and increase in MAPT expression after silencing GRN. c Increase in GRN expression is shown after knockdown of C9orf72 and MAPT 
However we also observed a decrease of C9orf72 in $M A P T$ patients, which would be consistent with a functional interaction.

To explore this possibility, we transduced $\mathrm{BE}(2) \mathrm{M} 17$ human neuroblastoma cells with lentiviral constructs expressing shRNAs targeting C9orf72 transcripts 2 and 3, $M A P T$ and GRN. qPCR experiments on cDNAs from the transduced cells showed a clear influence on expression of C9orf72 transcripts 2 and 3 after knock down of $M A P T$ and GRN separately compared to scrambled controls (Fig. 7). In particular we observed a reduction of total C9orf72 and transcript 2 and 3 when knocking down $M A P T$, while down regulation of GRN led to increased expression of C9orf72. Moreover C9orf72 knock down revealed a decrease in MAPT expression and an increase of GRN expression, endorsing the hypothesis that MAPT, GRN and C9orf72 interact in an a yet unknown common pathway.

\section{C9orf72 reduction and abundant C9orf72 antisense RNA foci in CD14+ monocytes of C9orf72-HRE patients} Our analysis of global C9orf72 expression using CAGEseq data across the FANTOM5 collection showed that C9orf72 is highly expressed in myeloid cells, particularly in CD14+ monocytes, in agreement with previous findings based on microarrays [54].

We performed digital PCR experiments on cDNA from CD14+ monocytes and brain RNAs from control donors and confirmed the higher expression of C9orf72 in CD14+ monocytes (up to seven fold increase) as compared to brain (Additional file 1: Figure S7).

To investigate if the reduction of C9orf72 observed in post mortem brain tissue of FTD cases could also be observed in monocytes, we isolated CD14+ monocytes from control donors, C9orf72-HRE and non familial FTD, ALS and PD patients (Table 2).
qPCR experiments on cDNAs from patients and controls for all the C9orf72 transcripts showed a clear reduction of total C9orf72, transcripts 1 and 2 and AS1 in all the patients, as compared to controls (Additional file 1: Figure S9A, 9B and $9 \mathrm{C}$ and data not shown). In contrast transcript 3 was slightly increased in the C9orf72HRE cases (Additional file 1: Figure S9D). We did not detect differences in the expression of AS2, AS3 and TSS -S4 (data not shown).

Next we investigated if RNA foci were also present in CD14+ monocytes from C9orf72-HRE carriers. Interestingly, we could detect abundant RNA foci for the antisense C9orf72 (Fig. 8a and Table 3) with $80-90 \%$ of cells showing at least one nuclear focus and a small proportion of cells containing more than 20 foci. We observed RNA foci for the sense C9orf72 as well, at least one nuclear RNA focus in up to $29 \%$ of cells (Fig. 8b and Table 3).

Overall the percentage of RNA foci for the antisense C9orf72 in CD14+ monocytes was much higher (80$90 \%)$ than the percentage reported for peripheral blood leukocytes (7\%) [12] and for CNS (from 9 to $26 \%$ depending on the region) [6], while the number of RNA foci for the sense C9orf72 is rather similar to that in the CNS $[1,5,6,47,55]$, despite the fact C9orf 72 is much more abundant in CD14+ monocytes.

\section{Discussion}

Our analysis on global C9orf72 expression by using CAGEseq data reveals new and interesting features for the C9orf72 promoter and gene locus. A major strength of CAGEseq lies in its ability to distinguish closely spaced TSS usage preference that is often tissue or cell specific. Indeed we observed several novel TSSs at the C9orf72 locus with distinct modes of expression in a subset of myeloid cells and CNS suggesting they have a cell and/or tissue specific function. Interestingly,
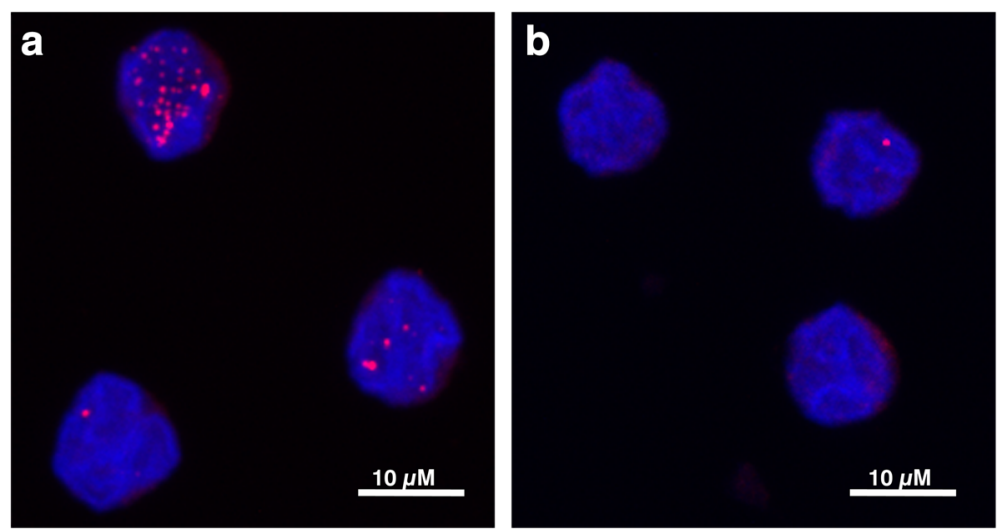

Fig. 8 RNA foci in C9orf72-HRE CD14+ monocytes. a Antisense foci in CD14+ monocytes from the same patient detected using a TYE563-conjugated FISH probe with sequence (GGGGCC)3. b Sense foci in CD14+ monocytes from a C9orf72-HRE patient detected with a TYE563-conjugated FISH probe with sequence (CCCCGG)3. Occasionally, antisense and sense foci were observed in the same cells; however they never co-localized 
Table 3 Summary of the RNA foci in CD14+ monocytes in C9orf72-HRE cases

\begin{tabular}{|c|c|c|c|c|c|c|}
\hline & Patient 1 SF & Patient 2 SF & Patient 3 SF & Patient 1 ASF & Patient 2 ASF & Patient 3 ASF \\
\hline Average & 0.3 & 0.5 & 0.5 & 5.5 & 8.5 & 7.8 \\
\hline Median & 0 & 0 & 0 & 3 & 4 & 4 \\
\hline Minimum & 0 & 0 & 0 & 0 & 0 & 0 \\
\hline Maximum & 10 & 4 & 6 & 25 & 38 & 35 \\
\hline$\%$ & 17 & 29 & 25 & 90 & 79 & 85 \\
\hline$n$ & 72 & 75 & 106 & 70 & 132 & 131 \\
\hline
\end{tabular}

The table summarizes sense and antisense foci counts from 3 patients with C9orf72-HRE

SF RNA sense foci, ASF RNA antisense foci

$\%=$ Percent of cells having at least one focus. No foci were detected in monocytes from an FTD patient without the repeat expansion

$\mathrm{N}=$ number of cells in which foci were counted automatically with a Cell Profiler speckle-counting algorithm

expression levels of S1 + S3b TSS for C9orf72 transcripts 1 and 3 show a high correlation with the IPO7 gene encoding for the importin 7 protein, a member of the $\beta$ karyopherin family proteins that mediate nuclear import of ribosomal proteins and export of ribosomal subunits necessary for ribosome biogenesis [44]. Currently we cannot predict whether the C9orf72-IPO7 correlation reflects a functional interaction, but it has been shown that C9orf72 protein isoforms localize to the nuclear membrane in healthy neurons and they interact with components of the nuclear pore complex [56]. It is therefore tempting to speculate that $C 9$ orf 72 transcripts 1 and 3 in the CNS might be physiologically involved in nuclear import and export that would be affected by a decrease in their expression. This potential detrimental effect could be exacerbated in C9orf72-HRE patients by the presence of HRE-containing transcripts, as indicated by several studies highlighting defects in nucleocytoplasmic trafficking as results of HRE expression [10, 11, 57]. HRE expression would additionally mediate a toxic gain of function by binding and sequestering proteins involved in nucleocytoplasmic trafficking.

More research is therefore warranted in characterizing C9orf72 functions in CNS, particularly when considering therapeutic efforts aiming at simply decreasing C9orf 72 expression to ameliorate RNA foci and DPR proteins aggregates formation.

Our CAGEseq data and qPCR results showed a consistent decrease in C9orf72 expression in C9orf72-HRE patients but also in MAPT and GRN mutations carriers pointing to additional regulatory mechanisms besides the HRE-mediated C9orf72 repression. On control brains we observed an association of the C9orf72-HRE risk haplotype with an increase in expression of $C 9$ orf 72 TSS for transcript 1 and 3 and decrease in TSS for transcript 2. The association of C9orf72 expression with SNPs in the risk haplotype has been already reported in monocytes but never in brain $[58,59]$. In our qPCR experiments we could detect separately C9orf 72 transcripts 1 and 3 and we replicated the association of the risk haplotype with the increase of C9orf 72 transcript 3 but not with transcript 1 or 2 , likely because of the small effect and sample size. At first glance the increased expression of transcript 3 determined by the risk haplotype appears to be in contradiction with the CAGEseq and qPCR expression data showing general reduction of transcript 3 in the C9orf72-HRE and FTD cases with $M A P T$ and GRN mutations compared with controls. However the increase in expression of C9orf72 transcript 3 in individuals carrying the risk haplotype is small. Additionally, approximately half of the GRN and MAPT samples do not carry the risk haplotype.

Albeit small, the increase in expression of C9orf72 transcript 3 might have consequences in C9orf72-HRE patients. The HRE can be transcribed in the pre-mRNA of abortive and/or mature C9orf72 transcripts 1 and 3 that would accumulate in RNA foci $[46,60]$. In this respect, any increase of transcripts 1 and 3 induced by the risk haplotype in C9orf72-HRE cases might intensify RNA foci formation although not necessarily DPR proteins aggregation as it has been suggested RAN translation might require transcripts containing the first entire intron as template [46].

The molecular reasons underlying the association of the risk haplotype with the increase in C9orf 72 transcript 3 expression are unknown. The C9orf72-HRE occurs on a risk haplotype covering $110 \mathrm{~kb}$ region between $M O B 3 B$ and C9orf72. The HRE is highly polymorphic and prone to expansion particularly in the context of the risk haplotype [3]. In addition it is adjacent to a GC-rich LCS containing a $10 \mathrm{bp}$ deletion that joins the repeat with the LCS region, which might promote the formation of hairpin secondary loop structures that impair DNA replication. The 10 bp deletion has been observed in up to $25 \%$ of $C 9$ orf72-HRE patients [51, 52]; however it was not present in any of the GRN and MAPT mutation carriers used in this study.

Therefore, additional mechanisms must play a role in regulating C9orf72 expression. We gathered convincing evidence for the existence of three distinct antisense transcripts head-to-head to C9orf72 that are highly expressed in myeloid cells and to a lower extent in brain 
tissues in C9orf72-HRE cases as well as in individual without the repeat expansion. Several C9orf72 antisense transcripts have been already detected in intron $1 \mathrm{~b}[12$, 15 ] and by using CAGEseq analysis we provide evidence for a longer antisense transcript that uses a more distal TSS and might extend through the HRE and accumulate in the RNA foci as shown by the slight increase in AS1 expression level by CAGEseq and qPCR in brains. The C9orf72-AS2 and AS3 transcripts are adjacent but downstream the HRE and they might be involved in C9orf72 regulation.

Gene regulation by antisense transcription is rather intriguing. Antisense transcripts are transcribed from the opposite strand of protein-coding genes. This genomic arrangement immediately suggests that the sense and antisense transcripts might act on each other and increasing evidence corroborates such an hypothesis [61]. Antisense transcription has been already related to C9orf72 regulation mainly in the context of the G-rich HRE that would mediate RNA-DNA hybrid formation (R-loops) facilitating RNA polymerase II pausing before efficient termination causing transcriptional stalling and nucleolar stress [17]. We observed a negative correlation between $C 9$ orf 72 sense transcripts and antisense transcript AS3 in brain tissues from C9orf72-HRE patients and therefore it might be relevant to investigate whether the novel antisense transcripts hamper or amplify the HRE antisense effects. In addition increasing evidence shows that antisense transcripts act at nearly every level of gene regulation (transcription, mRNA processing and translation) and even function simultaneously at multiple steps. It has been shown that antisense expression can regulate transcription by affecting DNA methylation. Several studies, including our own, show that hypermethylation of the CpG-island at the $5^{\prime}$ end of the repeat contributes to C9orf72 repression in a high of C9orf72-HRE carriers, yet down-regulation of $C 9$ orf72 is more prevalent and shown among FTD sporadic patients [19] and MAPT and GRN mutation carriers (this study). Very recently, colocalization of para-nucleolar DPR proteins inclusions with heterochomatin and H3K9me2 [18], a marker of transcriptional repression linked to R-loop-induced transcriptional silencing, has been reported [62]. In light of these findings it would be interesting to determine whether and to what extent the C9orf 72 antisense AS3 affects histone modifications at the C9orf 72 locus not only in C9orf72-HRE but also in MAPT and GRN patients.

The robust C9orf 72 expression in myeloid cells and the changes in C9orf72 expression in CD14+ monocytes after exposure to microbial pathogens suggest that C9orf72 might be involved in immune-related processes, in agreement also with the recent findings that in mice is required for normal function of myeloid cells [63]. Moreover it has been proposed that $C 9$ orf 72 might play a role in the initial steps of autophagy as suggested by its interaction with FIP200, a component of the ULK1ATG13 autophagosome initiation complex [64].

Consistent with a possible C9orf72 role in autophagy, neurons differentiated from patients-derived iPSC, are more sensitive to autophagy inhibitors and presented higher p62 levels suggesting autophagy might be compromised [47].

The half-life of monocytes in blood is relatively short: approximately 1 day in mice and 3 days in human. Once monocytes are stimulated by an inflammatory response they activate pro-survival pathways, migrate to tissues where they mediate direct antimicrobial activity by releasing tumor necrosis factor, chemokines, engage in phagocytosis and differentiate to macrophages through molecular mechanisms that involve autophagy [65].

While waiting for further research to clarify the exact role of C9orf72 in the autophagy process it is tempting to speculate that reduced $C 9$ orf 72 expression might impair autophagy affecting monocyte-macrophage differentiation and thereby hamper host inflammatory responses. Although our findings will need to be replicated in a larger samples size including age-matched controls, our qPCR results on $\mathrm{CD} 14+$ monocytes from C9orf7-HRE carriers and from patients with clinical diagnosis of FTD and ALS are in line with this hypothesis. Currently we do not have evidence that monocytes in these patients are impaired but we show that they carry a very high antisense RNA foci burden.

\section{Conclusion}

In conclusion, our findings strongly imply that several mechanisms acting independently from the HRE, or in a concerted manner, contribute to regulate C9orf72 expression. We showed that the decrease in C9orf72 expression is a widespread phenomenon in FTD pathogenesis suggesting C9orf 72 plays a more general role in neurodegeneration. Moreover the C9orf72 TSSs profile in CNS and myeloid cells suggests distinct C9orf72 transcripts have tissue specific function and they call attention to a potential $C$ orff2 role in immune response.

\section{Additional file}

Additional file 1: Supplementary Data. Figure S1: Definition of TSSs at the C9orf72 locus; Figure S2: C9orf72 expression changes in challenged CD14+ monocytes. Figure S3: Distinct TSSs expression at C9orf72 locus in CNS, myeloid and lymphoid cells. Figure S4: Sense and antisense C9orf72 transcripts at the C9orf72 locus. Figure S5: Distinct C9orf72 TSSs expression in control brains. Figure S6: C9orf72 expression in adult and fetal cortex. Figure S7: C9orf72 expression level in CD14+ monocytes, brain tissue and microglia. Figure S8: C9orf72 expression in brains of patients with different neurodegenerative diseases. Figure S9: C9orf72 expression in CD14+ monocytes. Suppl. excel File 1: WGCNA results. Suppl. excel File 2: Correlations values between C9orf72 TSS(s) and all the other TSSs in the co-expressed modules. Table S1: Expression of C9orf72 TSSs as defined in 
CAGEseq dataset 1. Table S2: Expression of C9orf72 TSSs as defined in CAGEseq dataset 2. Table S3: List of primers used in this study. Table S4: Biological functions significant to the three modules related to C9orf72 TSSs as identified by WGCNA. Table S5: Biological functions significant for genes that correlate with C9orf72 TSSs in the WGCNA identified modules. Table S6: Summary of the Mann-Whitney test performed on NRQ values from QPCR experiments on medial frontal gyrus. (ZIP $20675 \mathrm{~kb}$ )

\section{Competing interests}

The authors declare that they have no competing interests.

\section{Authors' Contribution}

PR: contribution to study design, manuscript draft, data acquisition and interpretation. CB and $\mathrm{SH}$ : data acquisition, analysis and interpretation, contribution to manuscript draft. EL: data acquisition and interpretation. AD, MCL, EP and JSS: data acquisition. MS and MH: selection of appropriate blood donors including clinical information. MF: data analysis and interpretation, contribution to manuscript draft. PH: study conception and design, manuscript revision regarding intellectual content. All authors read and approved the final manuscript.

\section{Acknowledgments}

The authors would like to thank the Netherlands Brain Bank for providing brain tissues from controls donors and patients. We also thank the Centre for Clinical Transfusion Medicine in Tübingen, for control donors. We also would like to thank all the patients for their valuable contributions. We wish to thank all the members of the FANTOM5 consortium for contributing to the generation of samples and analysis of datasets. This research was partly supported by the EU Joint Program-Neurodegenerative Diseases (JPND): RiMod-FTD (PH and MF).

\section{Author details}

${ }^{1}$ German Center for Neurodegenerative Diseases (DZNE), Otfried-Müller Strasse 23, Tübingen 72076, Germany. ${ }^{2}$ Department of Neurodegenerative Diseases, Hertie Institute for Clinical Brain Research, University of Tübingen, Tübingen 72076, Germany.

\section{Received: 25 March 2016 Accepted: 25 March 2016}

\section{Published online: 14 April 2016}

\section{References}

1. De Jesus-Hernandez M, Mackenzie IR, Boeve BF, Boxer AL, Baker M, Rutherford NJ, et al. Expanded GGGGCC hexanucleotide repeat in noncoding region of C9ORF72 causes chromosome 9p-linked FTD and ALS. Neuron. 2011;72:245-56. doi:10.1016/j.neuron.2011.09.011.

2. Renton AE, Majounie E, Waite A, Simon-Sanchez J, Rollinson S, Gibbs JR, et al. A hexanucleotide repeat expansion in C9ORF72 is the cause of chromosome 9p21-linked ALS-FTD. Neuron. 2011;72:257-68. doi:10.1016/j. neuron.2011.09.010.

3. Heutink $P$, Jansen IE, Lynes EM. C9orf72; abnormal RNA expression is the key. Exp Neurol. 2014;262(Pt B):102-10. doi:10.1016/j.expneurol.2014.05.020

4. Mizielinska S, Isaacs AM. C9orf72 amyotrophic lateral sclerosis and frontotemporal dementia: gain or loss of function? Curr Opin Neurol. 2014;27:515-23. doi:10.1097/WCO.0000000000000130.

5. Lagier-Tourenne C, Baughn M, Rigo F, Sun S, Liu P, Li HR, et al. Targeted degradation of sense and antisense C9orf72 RNA foci as therapy for ALS and frontotemporal degeneration. Proc Natl Acad Sci U S A. 2013;110:E4530-9. doi:10.1073/pnas.1318835110.

6. Mizielinska S, Lashley T, Norona FE, Clayton EL, Ridler CE, Fratta P, et al. C9orf72 frontotemporal lobar degeneration is characterised by frequent neuronal sense and antisense RNA foci. Acta Neuropathol. 2013;126:845-57. doi:10.1007/s00401-013-1200-z.

7. Fratta P, Poulter M, Lashley T, Rohrer JD, Polke JM, Beck J, et al. Homozygosity for the C9orf72 GGGGCC repeat expansion in frontotemporal dementia. Acta Neuropathol. 2013;126:401-9. doi:10.1007/s00401-013-1147-0.

8. Zamiri B, Reddy K, Macgregor Jr RB, Pearson CE. TMPyP4 porphyrin distorts RNA G-quadruplex structures of the disease-associated r(GGGGCC)n repeat of the C9orf72 gene and blocks interaction of RNA-binding proteins. J Biol Chem. 2014;289:4653-9. doi:10.1074/jbc.C113.502336.
9. Prudencio M, Belzil W, Batra R, Ross CA, Gendron TF, Pregent LJ, et al. Distinct brain transcriptome profiles in C9orf72-associated and sporadic ALS Nat Neurosci. 2015;18:1175-82. doi:10.1038/nn.4065.

10. Zhang K, Donnelly CJ, Haeusler AR, Grima JC, Machamer JB, Steinwald P, et al. The C9orf72 repeat expansion disrupts nucleocytoplasmic transport. Nature. 2015;525:56-61. doi:10.1038/nature14973.

11. Freibaum BD, Lu Y, Lopez-Gonzalez R, Kim NC, Almeida S, Lee KH, et al. GGGGCC repeat expansion in C9orf72 compromises nucleocytoplasmic transport. Nature. 2015;525:129-33. doi:10.1038/nature14974.

12. Zu T, Liu Y, Banez-Coronel M, Reid T, Pletnikova O, Lewis J, et al. RAN proteins and RNA foci from antisense transcripts in C9ORF72 ALS and frontotemporal dementia. Proc Natl Acad Sci U S A. 2013;110:E4968-77. doi:10.1073/pnas.1315438110.

13. Mori K, Weng SM, Arzberger T, May S, Rentzsch K, Kremmer E, et al. The C9orf72 GGGGCC repeat is translated into aggregating dipeptide-repeat proteins in FTLD/ALS. Science. 2013;339:1335-8. doi:10.1126/science.1232927.

14. Ash PE, Bieniek KF, Gendron TF, Caulfield T, Lin WL, Dejesus-Hernandez M, et al. Unconventional translation of C9ORF72 GGGGCC expansion generates insoluble polypeptides specific to c9FTD/ALS. Neuron. 2013;77:639-46. doi:10.1016/j.neuron.2013.02.004.

15. Mori K, Arzberger T, Grasser FA, Gijselinck I, May S, Rentzsch K, et al. Bidirectional transcripts of the expanded C9orf72 hexanucleotide repeat are translated into aggregating dipeptide repeat proteins. Acta Neuropathol. 2013;126:881-93. doi:10.1007/s00401-013-1189-3.

16. Gendron TF, Bieniek KF, Zhang YJ, Jansen-West K, Ash PE, Caulfield T, et al. Antisense transcripts of the expanded C9ORF72 hexanucleotide repeat form nuclear RNA foci and undergo repeat-associated non-ATG translation in C9FTD/ALS. Acta Neuropathol. 2013;126:829-44. doi:10.1007/s00401-013-1192-8.

17. Haeusler AR, Donnelly CJ, Periz G, Simko EA, Shaw PG, Kim MS, et al. C9orf72 nucleotide repeat structures initiate molecular cascades of disease. Nature. 2014:507:195-200. doi:10.1038/nature13124.

18. Schludi MH, May S, Grasser FA, Rentzsch K, Kremmer E, Kupper C, et al. Erratum to: Distribution of dipeptide repeat proteins in cellular models and C9orf72 mutation cases suggests link to transcriptional silencing. Acta Neuropathol. 2015;130:557-8. doi:10.1007/s00401-015-1464-6.

19. Ciura S, Lattante $S$, Le Ber I, Latouche M, Tostivint $H$, Brice $A$, et al. Loss of function of C9orf72 causes motor deficits in a zebrafish model of amyotrophic lateral sclerosis. Ann Neurol. 2013;74:180-7. doi:10.1002/ana.23946.

20. Therrien M, Rouleau GA, Dion PA, Parker JA. Deletion of C9ORF72 results in motor neuron degeneration and stress sensitivity in C. elegans. PLoS One. 2013:8:e83450. doi:10.1371/journal.pone.0083450.

21. Koppers M, Blokhuis AM, Westeneng HJ, Terpstra ML, Zundel CA, Vieira de Sa R, et al. C9orf72 ablation in mice does not cause motor neuron degeneration or motor deficits. Ann Neurol. 2015;78:426-38. doi:10.1002/ana.24453.

22. Rubio-Texeira M, Kaiser CA. Amino acids regulate retrieval of the yeast general amino acid permease from the vacuolar targeting pathway. Mol Biol Cell. 2006:17:3031-50. doi:10.1091/mbc.E05-07-0669.

23. Zhang D, lyer LM, He F, Aravind L. Discovery of Novel DENN Proteins: Implications for the Evolution of Eukaryotic Intracellular Membrane Structures and Human Disease. Front Genet. 2012;3:283. doi:10.3389/ fgene.2012.00283.

24. Levine TP, Daniels RD, Gatta AT, Wong LH, Hayes MJ. The product of C9orf72, a gene strongly implicated in neurodegeneration, is structurally related to DENN Rab-GEFs. Bioinformatics. 2013;29:499-503. doi:10.1093/ bioinformatics/bts725.

25. Farg MA, Sundaramoorthy V, Sultana JM, Yang S, Atkinson RA, Levina V, et al. C9ORF72, implicated in amytrophic lateral sclerosis and frontotemporal dementia, regulates endosomal trafficking. Hum Mol Genet. 2014;23:3579-95. doi:10.1093/hmg/ddu068.

26. Xi Z, Zinman L, Moreno D, Schymick J, Liang Y, Sato C, et al. Hypermethylation of the CpG island near the G4C2 repeat in ALS with a C9orf72 expansion. Am J Hum Genet. 2013;92:981-9. doi:10.1016/j.ajhg.2013.04.017.

27. Xi Z, Rainero I, Rubino E, Pinessi L, Bruni AC, Maletta RG, et al. Hypermethylation of the CpG-island near the C9orf72 G(4)C(2)-repeat expansion in FTLD patients. Hum Mol Genet. 2014;23:5630-7. doi:10.1093/hmg/ddu279.

28. Consortium F, the RP, Clst, Forrest AR, Kawaji H, Rehli M, et al. A promoter level mammalian expression atlas. Nature. 2014;507:462-70. doi:10.1038/ nature13182. 
29. Severin J, Lizio M, Harshbarger J, Kawaji H, Daub CO, Hayashizaki Y, et al. Interactive visualization and analysis of large-scale sequencing datasets using ZENBU. Nat Biotechnol. 2014;32:217-9. doi:10.1038/nbt.2840.

30. Takahashi H, Lassmann T, Murata M, Carninci P. 5' end-centered expression profiling using cap-analysis gene expression and next-generation sequencing. Nat Protoc. 2012;7:542-61. doi:10.1038/nprot.2012.005.

31. Lassmann T, Hayashizaki Y, Daub CO. TagDust-a program to eliminate artifacts from next generation sequencing data. Bioinformatics. 2009;25:2839-40. doi:10.1093/bioinformatics/btp527.

32. Li H, Durbin R. Fast and accurate short read alignment with Burrows-Wheeler transform. Bioinformatics. 2009;25:1754-60. doi:10.1093/bioinformatics/btp324.

33. Harbers M, Kato S, de Hoon M, Hayashizaki Y, Carninci P, Plessy C. Comparison of RNA- or LNA-hybrid oligonucleotides in template-switching reactions for high-speed sequencing library preparation. BMC Genomics. 2013;14:665. doi:10.1186/1471-2164-14-665.

34. Langfelder P, Horvath S. WGCNA: an R package for weighted correlation network analysis. BMC Bioinformatics. 2008;9:559. doi:10.1186/1471-2105-9-559.

35. Chung DW, Rudnicki DD, Yu L, Margolis RL. A natural antisense transcript at the Huntington's disease repeat locus regulates HTT expression. Hum Mol Genet. 2011;20:3467-77. doi:10.1093/hmg/ddr263.

36. Hellemans J, Mortier G, De Paepe A, Speleman F, Vandesompele J. qBase relative quantification framework and software for management and automated analysis of real-time quantitative PCR data. Genome Biol. 2007:8:R19. doi:10.1186/gb-2007-8-2-r19.

37. Waite AJ, Baumer D, East S, Neal J, Morris HR, Ansorge O, et al. Reduced C9orf72 protein levels in frontal cortex of amyotrophic lateral sclerosis and frontotemporal degeneration brain with the C9ORF72 hexanucleotide repeat expansion. Neurobiol Aging. 2014;35:1779. doi:10.1016/j. neurobiolaging.2014.01.016. e1775-1779 e1713.

38. Mok K, Traynor BJ, Schymick J, Tienari PJ, Laaksovirta H, Peuralinna T, et al. Chromosome 9 ALS and FTD locus is probably derived from a single founder. Neurobiol Aging. 2012;33(209):e203-8. doi:10.1016/j. neurobiolaging.2011.08.005.

39. Shabalin AA. Matrix eQTL: ultra fast eQTL analysis via large matrix operations. Bioinformatics. 2012;28:1353-8. doi:10.1093/bioinformatics/bts163.

40. Russ J, Liu EY, Wu K, Neal D, Suh E, Irwin DJ, et al. Hypermethylation of repeat expanded C9orf72 is a clinical and molecular disease modifier. Acta Neuropathol. 2015;129:39-52. doi:10.1007/s00401-014-1365-0.

41. Moffat J, Grueneberg DA, Yang X, Kim SY, Kloepfer AM, Hinkle G, et al. A lentiviral RNAi library for human and mouse genes applied to an arrayed viral high-content screen. Cell. 2006;124:1283-98. doi:10.1016/ j.cell.2006.01.040.

42. Carpenter $\mathrm{AE}$, Jones TR, Lamprecht MR, Clarke C, Kang $\mathrm{H}$, Friman O, et al. Cell Profiler: image analysis software for identifying and quantifying cell phenotypes. Genome Biol. 2006;7:R100. doi:10.1186/gb-2006-7-10-r100.

43. Schindelin J, Arganda-Carreras I, Frise E, Kaynig V, Longair M, Pietzsch T, et al. Fiji: an open-source platform for biological-image analysis. Nat Methods. 2012;9:676-82. doi:10.1038/nmeth.2019.

44. Golomb L, Bublik DR, Wilder S, Nevo R, Kiss V, Grabusic K, et al. Importin 7 and exportin 1 link c-Myc and p53 to regulation of ribosomal biogenesis. Mol Cell. 2012;45:222-32. doi:10.1016/j.molcel.2011.11.022

45. Kang HJ, Kawasawa Yl, Cheng F, Zhu Y, Xu X, Li M, et al. Spatio-temporal transcriptome of the human brain. Nature. 2011:478:483-9. doi:10.1038/nature10523.

46. van Blitterswijk M, Gendron TF, Baker MC, DeJesus-Hernandez M, Finch NA, Brown PH, et al. Novel clinical associations with specific C9ORF72 transcripts in patients with repeat expansions in C9ORF72. Acta Neuropathol. 2015;130:863-76. doi:10.1007/s00401-015-1480-6.

47. Almeida S, Gascon E, Tran H, Chou HJ, Gendron TF, Degroot S, et al. Modeling key pathological features of frontotemporal dementia with C9ORF72 repeat expansion in iPSC-derived human neurons. Acta Neuropathol. 2013;126:385-99. doi:10.1007/s00401-013-1149-y.

48. Belzil W, Bauer PO, Prudencio M, Gendron TF, Stetler CT, Yan IK, et al. Reduced C9orf72 gene expression in C9FTD/ALS is caused by histone trimethylation, an epigenetic event detectable in blood. Acta Neuropathol. 2013;126:895-905. doi:10.1007/s00401-013-1199-1.

49. Donnelly CJ, Zhang PW, Pham JT, Haeusler AR, Mistry NA, Vidensky S, et al. RNA toxicity from the ALS/FTD C9ORF72 expansion is mitigated by antisense intervention. Neuron. 2013;80:415-28. doi:10.1016/j.neuron. 2013.10.015.

50. Gijselinck I, Van Langenhove T, van der Zee J, Sleegers K, Philtjens S, Kleinberger $G$, et al. A C9orf72 promoter repeat expansion in a Flanders-Belgian cohort with disorders of the frontotemporal lobar degeneration-amyotrophic lateral sclerosis spectrum: a gene identification study. Lancet Neurol. 2012;11:54-65. doi:10.1016/S1474-4422(11)70261-7.

51. van der Zee J, Gijselinck I, Dillen L, Van Langenhove T, Theuns J, Engelborghs S, et al. A pan-European study of the C9orf72 repeat associated with FTLD: geographic prevalence, genomic instability, and intermediate repeats. Hum Mutat. 2013;34:363-73. doi:10.1002/humu.22244.

52. Rollinson S, Bennion Callister J, Young K, Ryan SJ, Druyeh R, Rohrer JD, et al. A small deletion in C9orf72 hides a proportion of expansion carriers in FTLD. Neurobiol Aging. 2015;36(1601):e1601-5. doi:10.1016/j.neurobiolaging. 2014.12.009.

53. Xi Z, Zhang M, Bruni AC, Maletta RG, Colao R, Fratta P, et al. The C9orf72 repeat expansion itself is methylated in ALS and FTLD patients. Acta Neuropathol. 2015;129:715-27. doi:10.1007/s00401-015-1401-8.

54. Nataf S, Pays L. Gene co-expression analysis unravels a link between C9orf72 and RNA metabolism in myeloid cells. Acta Neuropathol Commun. 2015;3:64. doi:10.1186/s40478-015-0242-y.

55. Sareen D, O'Rourke JG, Meera P, Muhammad AK, Grant S, Simpkinson M, et al. Targeting RNA foci in iPSC-derived motor neurons from ALS patients with a C9ORF72 repeat expansion. Sci Transl Med. 2013;5:208ra. doi:10.1126/ scitransImed.3007529. 149.

56. Xiao S, MacNair L, McGoldrick P, McKeever PM, McLean JR, Zhang M, et al. Isoform-specific antibodies reveal distinct subcellular localizations of C9orf72 in amyotrophic lateral sclerosis. Ann Neurol. 2015;78:568-83. doi:10.1002/ana.24469.

57. Jovicic A, Mertens J, Boeynaems S, Bogaert E, Chai N, Yamada SB, et al. Modifiers of C9orf72 dipeptide repeat toxicity connect nucleocytoplasmic transport defects to FTD/ALS. Nat Neurosci. 2015;18:1226-9. doi:10.1038/nn.4085.

58. Zeller T, Wild P, Szymczak S, Rotival M, Schillert A, Castagne R, et al. Genetics and beyond-the transcriptome of human monocytes and disease susceptibility. PLoS One. 2010;5:e10693. doi:10.1371/journal.pone.0010693.

59. Diekstra FP, Saris CG, van Rheenen W, Franke $L$, Jansen RC, van Es MA, et al. Mapping of gene expression reveals CYP27A1 as a susceptibility gene for sporadic ALS. PLoS One. 2012;7:e35333. doi:10.1371/journal.pone.0035333.

60. Niblock M, Smith BN, Lee YB, Sardone V, Topp S, Troakes C, et al. Retention of hexanucleotide repeat-containing intron in C9orf72 mRNA: implications for the pathogenesis of ALS/FTD. Acta Neuropathol Commun. 2016;4:18. doi:10.1186/s40478-016-0289-4.

61. Pelechano V, Steinmetz LM. Gene regulation by antisense transcription. Nat Rev Genet. 2013;14:880-93. doi:10.1038/nrg3594.

62. Skourti-Stathaki K, Kamieniarz-Gdula K, Proudfoot NJ. R-loops induce repressive chromatin marks over mammalian gene terminators. Nature. 2014:516:436-9. doi:10.1038/nature13787.

63. O'Rourke JG, Bogdanik L, Yanez A, Lall D, Wolf AJ, Muhammad AK, et al. C9orf72 is required for proper macrophage and microglial function in mice. Science. 2016;351:1324-9. doi:10.1126/science.aaf1064.

64. Behrends C, Sowa ME, Gygi SP, Harper JW. Network organization of the human autophagy system. Nature. 2010;466:68-76. doi:10.1038/nature09204.

65. Zhang Y, Morgan MJ, Chen K, Choksi S, Liu ZG. Induction of autophagy is essential for monocyte-macrophage differentiation. Blood. 2012;119:2895-905. doi:10.1182/blood-2011-08-372383.

\section{Submit your next manuscript to BioMed Central and we will help you at every step:}

- We accept pre-submission inquiries

- Our selector tool helps you to find the most relevant journal

- We provide round the clock customer support

- Convenient online submission

- Thorough peer review

- Inclusion in PubMed and all major indexing services

- Maximum visibility for your research

Submit your manuscript at www.biomedcentral.com/submit 